DRAFT VERSION MAY 27, 2022

Preprint typeset using LTEX style emulateapj v. 12/16/11

\title{
KINEMATICS OF THE OVI CIRCUMGALACTIC MEDIUM: HALO MASS DEPENDENCE AND OUTFLOW SIGNATURES
}

\author{
Mason NG ${ }^{1,2, \dagger}$, Nikole M. Nielsen ${ }^{1,3}$, Glenn G. KaCPrZak ${ }^{1,3}$, Stephanie K. Pointon $^{1,3}$, Sowgat MuZahid ${ }^{4,5}$, \\ Christopher W. ChurChill ${ }^{6}$, AND JANe C. Charlton ${ }^{4}$, \\ ${ }^{1}$ Centre for Astrophysics and Supercomputing, Swinburne University of Technology, Hawthorn, Victoria 3122, Australia \\ ${ }^{2}$ Research School of Astronomy and Astrophysics, Australian National University, ACT 2611, Australia \\ ${ }^{3}$ ARC Centre of Excellence for All Sky Astrophysics in 3 Dimensions (ASTRO 3D), Australia \\ ${ }^{4}$ Department of Astronomy \& Astrophysics, The Pennsylvania State University, State College, PA 16801, USA \\ ${ }^{5}$ Leiden Observatory, Leiden University, PO Box 9513, NL-2300 RA Leiden, The Netherlands \\ ${ }^{6}$ Department of Astronomy, New Mexico State University, Las Cruces, NM 88003, USA \\ Draft version May 27, 2022
}

\begin{abstract}
We probe the high-ionization circumgalactic medium by examining absorber kinematics, absorber-galaxy kinematics, and average absorption profiles of 31 OVI absorbers from the "Multiphase Galaxy Halos" Survey as a function of halo mass, redshift, inclination, and azimuthal angle. The galaxies are isolated at $0.12<z_{\text {gal }}<$ 0.66 and are probed by a background quasar within $D \approx 200 \mathrm{kpc}$. Each absorber-galaxy pair has Hubble Space Telescope images and COS quasar spectra, and most galaxy redshifts have been accurately measured from Keck/ESI spectra. Using the pixel-velocity two-point correlation function (TPCF) method, we find that O VI absorber kinematics have a strong halo mass dependence. Absorbers hosted by $\sim L^{*}$ galaxies have the largest velocity dispersions, which we interpret to be that the halo virial temperature closely matches the temperature at which the collisionally ionized OVI fraction peaks. Lower mass galaxies and group environments have smaller velocity dispersions. Total column densities follow the same behavior, consistent with theoretical findings. After normalizing out the observed mass dependence, we studied absorber-galaxy kinematics with a modified TPCF and found non-virialized motions due to outflowing gas. Edge-on minor axis gas has large optical depths concentrated near the galaxy systemic velocity as expected for bipolar outflows, while faceon minor axis gas has a smoothly decreasing optical depth distribution out to large normalized absorbergalaxy velocities, suggestive of decelerating outflowing gas. Accreting gas signatures are not observed due to "kinematic blurring" in which multiple line-of-sight structures are observed. These results indicate that galaxy mass dominates OVI properties over baryon cycle processes.
\end{abstract}

Subject headings: galaxies: halos — quasars: absorption lines

\section{INTRODUCTION}

The prodigious reserves of gas surrounding galaxies in the circumgalactic medium (CGM) play an important role in galaxy evolution (see review by Tumlinson et al. 2017). This gas is primarily derived from the intergalactic medium (IGM, e.g., Putman et al. 2012; Cooper et al. 2015; Glidden et al. 2016), from cannibalizing satellite galaxies (e.g., Cole et al. 2000; Cox et al. 2008; Qu et al. 2011; Lambas et al. 2012a,b; Kaviraj 2014; Ownsworth et al. 2014; Gómez-Guijarro et al. 2018), and from galactic feedback (e.g., Strickland \& Heckman 2009; Schaye et al. 2015; van de Voort 2017; Butler et al. 2017; Correa et al. 2018). The general accepted picture of how a typical galaxy evolves includes the accretion of relatively metal-poor gas from the CGM onto the galactic disk (see review by Kacprzak 2017), which is used to fuel star formation. Gas is then driven out of the galactic disk in outflows when massive stars explode as supernovae and produce metalenriched winds (e.g., Shen et al. 2012; Lehner et al. 2013; Ford et al. 2014; Muzahid et al. 2015). The velocities of the outflowing gas do not usually exceed the escape velocity of the galaxy (e.g., Tumlinson et al. 2011; Bouché et al. 2012; Stocke et al. 2013; Mathes et al. 2014; Bordoloi et al. 2014), thus the gas is recycled back onto the galaxy and could fuel further episodes of star formation (e.g., Oppenheimer et al. 2010; Ford et al. 2014; van de Voort 2017). This paints the

\footnotetext{
$\dagger$ masonng@mit.edu
}

picture of the baryon cycle within the galaxy virial radius.

The OVI $\lambda \lambda 1031,1037$ absorption doublet is a common tracer of the CGM, particularly in the high-temperature regime of $T \sim 10^{5} \mathrm{~K}$ (e.g., Prochaska et al. 2011; Tumlinson et al. 2011; Stocke et al. 2013; Savage et al. 2014; Churchill et al. 2015; Johnson et al. 2015; Kacprzak et al. 2015; Werk et al. 2016). Oppenheimer et al. (2016) employed the EAGLE simulations to investigate the presence and role of different oxygen species in the CGM, assuming that OVI is collisionally ionized. They found that $\mathrm{OVI}$ is not the dominant oxygen species in the CGM, and that the column densities for O VI peak for $L_{*}$ galaxies, while dropping for lower mass halos and group halos. This is thought to be due to the OVI ionization fraction strongly tracing the virial temperature of the galaxy, where the associated virial temperature for $L_{*}$ galaxies provides the optimal conditions for the presence of OVI. For less massive galaxies, the virial temperature would be too cool for strong OVI presence, whereas the virial temperature would be too high for group environments as a large fraction of OVI is ionized out to higher ionization species. Nelson et al. (2018) found similar trends in the OVI column density with halo mass, but attributed them to black hole feedback (also see Oppenheimer et al. 2018). Several other works have also shown this trend in both observations (Bielby et al. 2019; Zahedy et al. 2019) and with gaseous halo models (Qu \& Bregman 2018).

Using a sample of quasar absorption-line spectra from 
HST/COS identified as part of the "Multiphase Galaxy Halos" Survey, Kacprzak et al. (2015) found that OVI has an azimuthal angle preference, where OVI tends to reside along the projected major axis $\left(0^{\circ} \leq \Phi \leq 20^{\circ}\right)$ and/or along the projected minor axis $\left(60^{\circ} \leq \Phi \leq 90^{\circ}\right)$. They also found a very weak dependence of the $\mathrm{OVI}$ absorption on the galaxy inclination, where the covering fraction of the OVI gas is roughly constant over all inclination angles except for $i>70^{\circ}$, as the high inclination minimizes the geometrical cross-section of gas flows. Moreover, the mean equivalent widths of OVI in lower inclination $\left(i<45^{\circ}\right)$ galaxies and higher inclination $\left(i>45^{\circ}\right)$ galaxies are consistent with each other.

Previous kinematics studies examined the absorber velocity dispersions of OVI with pixel-velocity two-point correlation functions (TPCFs) to characterize the absorber velocity dispersions for isolated galaxies (Nielsen et al. 2017). The authors found that there was no dependence of OVI kinematics on the inclination angle, azimuthal angle, and/or galaxy color, which indirectly suggests a lack of dependence on current star formation activity. They attribute this to OVI absorbers having ample time to mix and form a kinematically uniform halo surrounding the galaxies. This is consistent with Ford et al. (2014), who found that O VI in simulations likely traces gas that originates from ancient outflows. These results are in contrast to $\mathrm{Mg}$ II kinematics, which depends strongly on galaxy color, redshift, inclination, and azimuthal angle (Nielsen et al. 2015, 2016).

Pointon et al. (2017) examined OVI kinematics using TPCFs for galaxy group environments and found that the OVI absorption profiles for galaxy group environments are narrower compared to isolated galaxies. They posit that the virial temperature of the CGM in galaxy group environments (with more massive halos) is hot enough to ionize a larger fraction of OVI to higher order species to result in a lower OVI ionization fraction compared to isolated galaxies, consistent with the findings of Oppenheimer et al. (2016). This suggests that halo mass needs to be considered when studying the absorber kinematics of OVI.

Focusing on OVI absorber-galaxy kinematics, Tumlinson et al. (2011) found that O VI absorber-galaxy velocities rarely exceed the host galaxy escape velocity, indicating that the gas is bound. Mathes et al. (2014) found similar results, but noted that the fraction of gas that exceeds host galaxy escape velocities decreases with increasing halo mass. The authors suggested that wind recycling is increasingly important as the halo mass increases, consistent with simulations (Oppenheimer et al. 2010). Most recently, Kacprzak et al. (2019) related OVI absorber kinematics to host galaxy rotation curves. They found that along the projected galaxy major axis, where accretion is expected, OVI does not correlate with galaxy rotation kinematics like Mg II (e.g., Steidel et al. 2002; Kacprzak et al. 2010, 2011; Ho et al. 2017). For gas observed along the projected galaxy minor axis, O VI absorbers best match models of decelerating outflows. Combined with simulations, the authors suggest that $\mathrm{OVI}$ is not an ideal probe of gas accretion or outflows, but rather traces the virial temperature of the host halo.

The work presented here will address both the halo mass dependence of OVI absorber kinematics, and how OVI gas flows relative to the host galaxies by examining the absorbergalaxy kinematics, using a subset of OVI absorbers from the "Multiphase Galaxy Halos" Survey. We employ two TPCF methods: (1) absorber kinematics, which is the approach employed by Nielsen et al. (2017), and (2) absorber-galaxy kine- matics. In constructing the TPCFs for absorber-galaxy kinematics (method 2), we apply the velocity offset between the absorber redshift and the galaxy redshift. We also normalize the absorber-galaxy velocities with respect to the circular velocity at the observed impact parameter, $V_{\mathrm{c}}(D)$, to take into consideration the range of halo masses in the sample (similar to the normalization done in Nielsen et al. 2016). Average absorption profiles are presented to complement the TPCFs by providing information about the optical depth.

In Section 2, we present the sample and elaborate on how the kinematics are quantified, namely with the TPCFs and average absorption profiles. In Section 3, we present the mass dependence of absorber kinematics, comparing our sample to the group environment sample published in Pointon et al. (2017) and the simulated aperture column densities presented by Oppenheimer et al. (2016). Section 4 presents new absorber-galaxy kinematics for various subsamples segregated by galaxy redshift, $z_{\text {gal }}$, inclination, $i$, azimuthal angle, $\Phi$, and halo mass, $\log \left(M_{\mathrm{h}} / M_{\odot}\right)$. In Section 5, we discuss the halo mass dependence of $\mathrm{OVI}$ absorber kinematics and non-virialized motions in the form of outflows. Finally, we conclude in Section 6. Throughout we assume a $\Lambda$ CDM cos$\operatorname{mology}\left(H_{0}=70 \mathrm{~km} \mathrm{~s}^{-1} \mathrm{Mpc}^{-1}, \Omega_{M}=0.3, \Omega_{\Lambda}=0.7\right)$.

\section{SAMPLE AND DATA ANALYSIS}

The sample of OVI absorber-galaxy pairs used in this work is a subset of the "Multiphase Galaxy Halos" Survey (Kacprzak et al. 2015, 2019; Muzahid et al. 2015, 2016; Nielsen et al. 2017; Pointon et al. 2017, 2019). The associated galaxy spectroscopic redshifts, $z_{\mathrm{gal}}$, spanning $0.1241 \leq$ $z_{\text {gal }} \leq 0.6610$ (median $\left\langle z_{\text {gal }}\right\rangle=0.2443$ ), are accurate to within $\sigma_{z} \leq 0.0001$, which is $\sim 30 \mathrm{~km} \mathrm{~s}^{-1}$ in velocity space (e.g., Kacprzak et al. 2019). The absorber-galaxy pairs are also within an on-the-sky projected distance (impact parameter) of $D \approx 200 \mathrm{kpc}(21.1 \mathrm{kpc}<D<276.3 \mathrm{kpc}$, median $\langle D\rangle=$ $93.2 \mathrm{kpc})$. All of the galaxies are isolated, that is, there were no identified neighboring galaxies within a projected distance of $200 \mathrm{kpc}$ from the line-of-sight of the quasar, and within a line-of-sight velocity separation of $500 \mathrm{~km} \mathrm{~s}^{-1}$. Absorption systems with line-of-sight velocities larger than $\pm 500 \mathrm{~km} \mathrm{~s}^{-1}$ away from their identified host galaxies are assumed not to be associated with the host galaxy. The sample is somewhat heterogeneous in that the quasar fields were drawn from several works (see Table 1 and Kacprzak et al. 2019) and each survey has different completeness levels. However, the quasar fields have generally been surveyed to a sensitivity of $0.1 L^{*}$ out to at least $350 \mathrm{kpc}$, with the exception of galaxies from the COSHalos survey, of which there are 12, which goes out to only $150 \mathrm{kpc}$ (for details, see Tumlinson et al. 2013; Werk et al. 2013).

For our OVI absorber kinematics analysis (Section 3), we also include a sample of six galaxy group environments from Pointon et al. (2017) for comparison. See Pointon et al. (2017) for further details. We include this sample to cover a large range in halo masses to investigate the OVI column density dependence on halo mass found in the EAGLE simulations (Oppenheimer et al. 2016).

\subsection{Galaxy Properties}

We have selected 31 absorber-galaxy pairs from the "Multiphase Galaxy Halos" Survey that are suitable for this study. Each galaxy in the sample was imaged with ACS, WFC3, or WFPC2 on the Hubble Space Telescope (HST). GIM2D 
Halo Mass Dependence of Ovi Kinematics

TABLE 1

Ovi Absorber-Galaxy Properties

\begin{tabular}{|c|c|c|c|c|c|c|c|c|c|c|c|}
\hline $\begin{array}{l}\text { (1) } \\
\text { Field }\end{array}$ & $\begin{array}{l}(2) \\
z_{\text {gal }} \\
\end{array}$ & $\begin{array}{l}(3) \\
\text { Ref }^{a} \\
\end{array}$ & $\begin{array}{l}\text { (4) } \\
z_{\text {abs }}\end{array}$ & $\begin{array}{c}(5) \\
W_{\mathrm{r}}(1031) \\
(\AA)\end{array}$ & $\begin{array}{c}(6) \\
\Phi \\
\text { (deg) }\end{array}$ & $\begin{array}{c}(7) \\
i \\
(\mathrm{deg})\end{array}$ & $\begin{array}{c}(8) \\
M_{r}{ }^{\mathrm{b}} \\
(\mathrm{AB})\end{array}$ & $\begin{array}{c}(9) \\
\log \left(M_{\mathrm{h}} / M_{\odot}\right)\end{array}$ & $\begin{array}{c}(10) \\
V_{\mathrm{c}}(D) \\
\left(\mathrm{km} \mathrm{s}^{-1}\right)\end{array}$ & $\begin{array}{c}(11) \\
D \\
(\mathrm{kpc})\end{array}$ & $\begin{array}{c}(12) \\
R_{\mathrm{vir}} \\
(\mathrm{kpc})\end{array}$ \\
\hline $\mathrm{J} 012528-000555$ & 0.398525 & 2 & 0.399090 & 0.817 & $73.4_{-4.7}^{+4.6}$ & $63.2_{-2.6}^{+1.7}$ & -21.99 & $12.51_{-0.15}^{+0.16}$ & $242.9_{-53.6}^{+68.8}$ & $163.0 \pm 0.1$ & $285.4_{-32.0}^{+37.3}$ \\
\hline J035128 - 142908 & 0.356992 & 1 & 0.356825 & 0.396 & $4.9_{-40.2}^{+33.0}$ & $28.5_{-12.5}^{+19.8}$ & -20.86 & $12.00_{-0.19}^{+0.29}$ & $174.8_{-50.6}^{+90.9}$ & $72.3 \pm 0.4$ & $190.8_{-25.9}^{+47.9}$ \\
\hline J040748 - 121136 & 0.3422 & 3 & 0.342042 & 0.056 & $48.1_{-0.9}^{+1.0}$ & $85.0_{-0.4}^{+0.1}$ & -19.77 & $11.62_{-0.21}^{+0.42}$ & $107.5_{-36.9}^{+83.8}$ & $172.0 \pm 0.1$ & $142.5_{-21.6}^{+53.9}$ \\
\hline J040748 - 121136 & 0.495164 & 4 & 0.495101 & 0.229 & $21.0_{-3.7}^{+5.3}$ & $67.2_{-7.5}^{+7.6}$ & -19.73 & $11.41_{-0.21}^{+0.45}$ & $97.5_{-33.7}^{+82.6}$ & $107.6 \pm 0.4$ & $124.4_{-18.2}^{+51.6}$ \\
\hline J045608 - 215909 & 0.381511 & 1 & 0.381514 & 0.219 & $63.8_{-2.7}^{+4.3}$ & $57.1_{-2.4}^{+19.9}$ & -20.87 & $12.00_{-0.19}^{+0.29}$ & $167.7_{-48.4}^{+86.8}$ & $103.4 \pm 0.3$ & $192.4_{-26.0}^{+48.0}$ \\
\hline $\mathrm{J} 091440+282330$ & 0.244312 & 1 & 0.244098 & 0.333 & $18.2_{-1.0}^{+1.1}$ & $39.0_{-0.2}^{+0.4}$ & -20.55 & $11.88_{-0.20}^{+0.33}$ & $153.2_{-46.9}^{+91.5}$ & $105.9 \pm 0.1$ & $170.7_{-23.9}^{+49.4}$ \\
\hline $\mathrm{J} 094331+053131$ & 0.353052 & 1 & 0.353286 & 0.220 & $8.2_{-5.0}^{+3.0}$ & $44.4_{-1.2}^{+1.1}$ & -19.88 & $11.66_{-0.21}^{+0.41}$ & $125.9_{-42.6}^{+95.4}$ & $96.5 \pm 0.3$ & $146.8_{-22.0}^{+54.1}$ \\
\hline J094331 + 053131 & 0.548494 & 1 & 0.548769 & 0.275 & $67.2_{-1.0}^{+0.9}$ & $58.8_{-1.1}^{+0.6}$ & -21.30 & $11.96_{-0.18}^{+0.26}$ & $150.3_{-41.3}^{+70.2}$ & $150.9 \pm 0.6$ & $190.9_{-24.9}^{+42.9}$ \\
\hline $\mathrm{J} 095000+483129$ & 0.211866 & 1 & 0.211757 & 0.211 & $16.6_{-0.1}^{+0.1}$ & $47.7_{-0.1}^{+0.1}$ & -21.73 & $12.37_{-0.16}^{+0.18}$ & $237.0_{-55.3}^{+74.7}$ & $93.6 \pm 0.2$ & $246.9_{-29.0}^{+36.1}$ \\
\hline $\mathrm{J} 100402+285535$ & 0.1380 & 5 & 0.137724 & 0.117 & $12.4_{-2.9}^{+2.4}$ & $79.1_{-2.1}^{+2.1}$ & -17.05 & $10.87_{-0.22}^{+0.63}$ & $69.9_{-27.6}^{+89.3}$ & $56.7 \pm 0.2$ & $76.3_{-11.7}^{+47.3}$ \\
\hline $\mathrm{J} 100902+071343$ & 0.227855 & 1 & 0.227851 & 0.576 & $89.6_{-1.3}^{+1.3}$ & $66.3_{-0.9}^{+0.6}$ & -20.19 & $11.76_{-0.21}^{+0.37}$ & $149.0_{-48.2}^{+101.2}$ & $64.0 \pm 0.8$ & $154.5_{-22.5}^{+50.9}$ \\
\hline $\mathrm{J} 104116+061016$ & 0.442173 & 1 & 0.441630 & 0.368 & $4.3_{-1.0}^{+0.9}$ & $49.8_{-5.2}^{+7.4}$ & -21.36 & $11.99_{-0.18}^{+0.26}$ & $173.8_{-47.1}^{+79.1}$ & $56.2 \pm 0.3$ & $193.1_{-24.9}^{+42.2}$ \\
\hline $\mathrm{J} 111908+211918$ & 0.1383 & 6 & 0.138521 & 0.074 & $34.4_{-0.4}^{+0.4}$ & $26.4_{-0.4}^{+0.8}$ & -21.45 & $12.24_{-0.17}^{+0.21}$ & $204.3_{-51.4}^{+76.5}$ & $138.0 \pm 0.2$ & $219.0_{-27.1}^{+38.8}$ \\
\hline $\mathrm{J} 113327+032719$ & 0.154599 & 4 & 0.153979 & 0.252 & $56.1_{-1.3}^{+1.7}$ & $23.5_{-0.2}^{+0.4}$ & -19.84 & $11.64_{-0.21}^{+0.41}$ & $139.8_{-47.5}^{+107.1}$ & $55.6 \pm 0.1$ & $138.7_{-20.9}^{+51.6}$ \\
\hline J113910 - 135043 & 0.204194 & 1 & 0.204297 & 0.231 & $5.8_{-0.5}^{+0.4}$ & $83.4_{-0.5}^{+0.4}$ & -19.99 & $11.69_{-0.21}^{+0.40}$ & $133.0_{-44.3}^{+98.0}$ & $93.2 \pm 0.3$ & $146.2_{-21.6}^{+52.4}$ \\
\hline J113910 - 135043 & 0.212259 & 1 & 0.212237 & 0.137 & $80.4_{-0.5}^{+0.4}$ & $85.0_{-0.6}^{+5.0}$ & -20.09 & $11.73_{-0.21}^{+0.39}$ & $119.2_{-39.2}^{+84.4}$ & $174.8 \pm 0.1$ & $150.3_{-22.0}^{+51.7}$ \\
\hline$J 113910-135043$ & 0.219724 & 4 & 0.219820 & 0.021 & $44.9_{-8.1}^{+8.9}$ & $85.0_{-8.5}^{+5.0}$ & -17.67 & $11.04_{-0.21}^{+0.60}$ & $66.8_{-25.8}^{+80.5}$ & $122.0 \pm 0.2$ & $88.7_{-13.5}^{+52.0}$ \\
\hline J113910 - 135043 & 0.319255 & 1 & 0.319167 & 0.255 & $39.1_{-1.7}^{+1.9}$ & $83.4_{-1.1}^{+1.4}$ & -20.48 & $11.86_{-0.20}^{+0.34}$ & $157.0_{-48.5}^{+96.1}$ & $73.3 \pm 0.4$ & $170.4_{-23.9}^{+50.7}$ \\
\hline $\mathrm{J} 121920+063838$ & 0.1241 & 6 & 0.124103 & 0.424 & $67.2_{-91.4}^{+39.8}$ & $22.0_{-21.8}^{+18.7}$ & -20.50 & $11.87_{-0.20}^{+0.34}$ & $156.8_{-48.4}^{+95.6}$ & $93.4 \pm 5.3$ & $163.1_{-22.9}^{+48.2}$ \\
\hline $\mathrm{J} 123304-003134$ & 0.318757 & 4 & 0.318609 & 0.439 & $17.0_{-2.3}^{+2.0}$ & $38.7_{-1.8}^{+1.6}$ & -20.62 & $11.91_{-0.20}^{+0.32}$ & $159.2_{-48.3}^{+92.5}$ & $88.9 \pm 0.2$ & $176.5_{-24.7}^{+49.7}$ \\
\hline $\mathrm{J} 124154+572107$ & 0.205267 & 1 & 0.205538 & 0.519 & $77.6_{-0.4}^{+0.3}$ & $56.4_{-0.5}^{+0.3}$ & -19.83 & $11.64_{-0.21}^{+0.41}$ & $145.0_{-49.3}^{+114.4}$ & $21.1 \pm 0.1$ & $140.2_{-21.1}^{+52.3}$ \\
\hline $\mathrm{J} 124154+572107$ & 0.217905 & 4 & 0.218043 & 0.366 & $63.0_{-2.1}^{+1.8}$ & $17.4_{-1.6}^{+1.4}$ & -19.77 & $11.62_{-0.21}^{+0.42}$ & $124.0_{-42.5}^{+96.7}$ & $94.6 \pm 0.2$ & $138.7_{-21.0}^{+52.4}$ \\
\hline $\mathrm{J} 124410+172104$ & 0.5504 & 5 & 0.550622 & 0.447 & $20.1_{-19.1}^{+16.7}$ & $31.7_{-4.8}^{+16.2}$ & -20.97 & $11.82_{-0.19}^{+0.31}$ & $144.4_{-42.3}^{+79.0}$ & $21.2 \pm 0.3$ & $171.2_{-23.1}^{+45.5}$ \\
\hline $\mathrm{J} 131956+272808$ & 0.6610 & 7 & 0.660670 & 0.311 & $86.6_{-1.2}^{+1.5}$ & $65.8_{-1.2}^{+1.2}$ & -21.70 & $12.15_{-0.15}^{+0.19}$ & $183.6_{-41.6}^{+59.8}$ & $103.9 \pm 0.5$ & $223.9_{-24.9}^{+34.7}$ \\
\hline $\mathrm{J} 132222+464546$ & 0.214431 & 1 & 0.214320 & 0.354 & $13.9_{-0.2}^{+0.2}$ & $57.9_{-0.2}^{+0.1}$ & -21.18 & $12.13_{-0.18}^{+0.25}$ & $204.0_{-54.7}^{+90.8}$ & $38.6 \pm 0.2$ & $204.6_{-26.1}^{+43.8}$ \\
\hline $\mathrm{J} 134251-005345$ & 0.227042 & 1 & 0.227196 & 0.373 & $13.2_{-0.4}^{+0.5}$ & $0.1_{-0.1}^{+0.6}$ & -21.77 & $12.39_{-0.16}^{+0.17}$ & $239.3_{-55.2}^{+73.7}$ & $35.3 \pm 0.2$ & $251.7_{-29.3}^{+35.9}$ \\
\hline $\mathrm{J} 155504+362847$ & 0.189201 & 1 & 0.189033 & 0.385 & $47.0_{-0.8}^{+0.3}$ & $51.8_{-0.7}^{+0.7}$ & -21.03 & $12.07_{-0.18}^{+0.27}$ & $196.3_{-54.2}^{+93.8}$ & $33.4 \pm 0.1$ & $193.7_{-25.2}^{+44.6}$ \\
\hline $\mathrm{J} 213135-120704$ & 0.4302 & 8 & 0.430164 & 0.385 & $14.9_{-4.9}^{+6.0}$ & $48.3_{-3.7}^{+3.5}$ & -21.47 & $12.04_{-0.18}^{+0.25}$ & $181.3_{-48.1}^{+79.0}$ & $48.4 \pm 0.2$ & $199.7_{-25.3}^{+41.8}$ \\
\hline $\mathrm{J} 225357+160853$ & 0.153718 & 9 & 0.153821 & 0.263 & $59.6_{-1.8}^{+0.9}$ & $33.3_{-2.0}^{+2.7}$ & -19.55 & $11.55_{-0.21}^{+0.45}$ & $137.8_{-48.1}^{+115.4}$ & $31.8 \pm 0.2$ & $129.5_{-19.6}^{+52.7}$ \\
\hline $\mathbf{J} 225357+160853$ & 0.352787 & 1 & 0.352708 & 0.381 & $88.7_{-4.8}^{+4.6}$ & $36.7_{-4.6}^{+6.9}$ & -20.67 & $11.93_{-0.20}^{+0.32}$ & $138.1_{-41.6}^{+78.4}$ & $203.2 \pm 0.5$ & $180.3_{-25.2}^{+49.5}$ \\
\hline $\mathrm{J} 225357+160853$ & 0.390013 & 9 & 0.390705 & 0.173 & $24.2_{-1.2}^{+1.2}$ & $76.1_{-1.2}^{+1.1}$ & -21.25 & $12.16_{-0.18}^{+0.24}$ & $160.4_{-42.5}^{+69.0}$ & $276.3 \pm 0.2$ & $217.2_{-27.5}^{+44.9}$ \\
\hline
\end{tabular}

a Galaxy redshift references: (1) Kacprzak et al. (2019), (2) Muzahid et al. (2015), (3) Johnson et al. (2013), (4) Pointon et al. (2019), (5) Chen et al. (2001), (6) Prochaska et al. (2011), (7) Kacprzak et al. (2012), (8) Guillemin \& Bergeron (1997), and (9) this work.

${ }^{\mathrm{b}}$ Galaxy absolute $r$-band magnitude in the AB system. The magnitudes are converted into Vega mags with $M_{r}-5 \log h=M_{r}(A B)-5 \log (0.7)-0.1429$, which are then used in the halo abundance matching method (for details, see Churchill et al. 2013).

(Simard et al. 2002) was then used to model the morphological properties of the galaxies; the details of the modeling are elaborated upon in Kacprzak et al. (2015). Following Nielsen et al. (2017), we define galaxies having inclination angles of $0^{\circ} \leq i<51^{\circ}$ as face-on, and galaxies with $51^{\circ} \leq i \leq 90^{\circ}$ as edge-on. We also define azimuthal angles of $0^{\circ} \leq \Phi<45^{\circ}$ as a quasar sightline aligned with the projected major axis of the galaxy, and azimuthal angles of $45^{\circ} \leq \Phi \leq 90^{\circ}$ as a quasar sightline aligned with the projected minor axis of the galaxy.

To measure accurate redshifts, galaxy spectra were obtained for a majority of our sample (24/31) using the Keck Echellette Spectrograph and Imager, ESI (Sheinis et al. 2002). Details of the observations and data reduction, and most of the new redshifts are presented in Kacprzak et al. (2019). Several additional redshifts determined with this method are pre- sented in Pointon et al. (2019) and here. The ESI spectra have a resolution of $22 \mathrm{~km} \mathrm{~s}^{-1}$ pixel $^{-1}(\mathrm{FWHM} \sim 90 \mathrm{~km} / \mathrm{s}$ ) when binned by two in the spectral direction and have a wavelength coverage of 4000 to $10000 \AA$, which allows for detection of multiple emission lines such as the [OII] doublet, $\mathrm{H} \beta$, [OIII] doublet, $\mathrm{H} \alpha$, and [N II] doublet. Galaxy spectra are both vacuum and heliocentric velocity-corrected to provide a direct comparison with the absorption line spectra. The Gaussian fitting algorithm FITTER (Churchill et al. 2000) was used to compute best-fit emission-line centroids and widths to derive galaxy redshifts. Galaxy redshifts obtained with ESI have accuracies ranging from $3-20 \mathrm{~km} \mathrm{~s}^{-1}$. To test for possible systematic shifts in our wavelength solutions that could increase our uncertainties, the derived wavelength solution was ver- 
ified against a catalog of known sky lines ranging between $4000-10000 \AA$. This test resulted in an rms difference of $\sim 0.03 \AA\left(\sim 2 \mathrm{~km} \mathrm{~s}^{-1}\right)$, which is lower than the galaxy redshift error obtained by fitting multiple emission and absorption lines. The remainder of the galaxy redshifts were obtained from previous studies, and are tabulated in Table 1 (Guillemin \& Bergeron 1997; Chen et al. 2001; Prochaska et al. 2011; Kacprzak et al. 2012; Johnson et al. 2013).

Since previous work has suggested that halo mass is key to governing the presence or absence of OVI absorbing gas (e.g., Oppenheimer et al. 2016; Pointon et al. 2017), we calculate halo masses, circular velocities, and virial radii for each of the galaxies in our sample to investigate this mass dependence for gas kinematics. We follow the halo abundance matching method described in Appendix A of Churchill et al. (2013) and summarize it here (also see Trujillo-Gomez et al. 2011). Halo abundance matching makes the assumption that the number density of galaxies with a given observed galaxy property (in this case, the COMBO-17 $r$-band luminosity functions from Wolf et al. 2003) is mapped to the distribution function of simulated galaxies with a given property (the maximum circular velocity or dark matter halo mass from the Bolshoi $N$-body cosmological simulation dark matter halo catalogs; Klypin et al. 2011). For each galaxy in our sample, the $r$-band absolute Vega magnitude, $M_{r}-5 \log (\mathrm{h})$, was calculated and used in the corresponding redshift curves from Figure 9(b) of Churchill et al. (2013) to calculate halo masses, $\log \left(M_{\mathrm{h}} / M_{\odot}\right)$. From the halo masses, we calculate the virial radii according to Bryan \& Norman (1998). Circular velocities, $V_{\mathrm{c}}(D)$, were calculated at the impact parameter of absorption using Equations 5 of Navarro et al. (1996) and B2 of Churchill et al. (2013). In the sample, the halo masses $\operatorname{span} 10.87 \leq \log \left(M_{\mathrm{h}} / M_{\odot}\right) \leq 12.51\left(\right.$ median $\left\langle\log \left(M_{\mathrm{h}} / M_{\odot}\right)\right\rangle=$ $11.88)$; the circular velocities span $66.8 \mathrm{~km} \mathrm{~s}^{-1} \leq V_{\mathrm{c}}(D) \leq$ $242.9 \mathrm{~km} \mathrm{~s}^{-1}$ (median $\left\langle V_{\mathrm{c}}(D)\right\rangle=150.3 \mathrm{~km} \mathrm{~s}^{-1}$ ).

In Table 1, we list the absorber-galaxy pairs used in this work, the corresponding galaxy redshifts, $z_{\text {gal }}$, O vI absorber redshifts, $z_{\mathrm{abs}}$, rest-frame equivalent widths, $W_{\mathrm{r}}(1031)$, azimuthal angle, $\Phi$, galaxy inclination, $i$, absolute $r$-band (AB) magnitude, $M_{r}$, halo mass, $\log \left(M_{\mathrm{h}} / M_{\odot}\right)$, circular velocity at the observed impact parameter, $V_{\mathrm{c}}(D)$, impact parameter, $D$, and the virial radius, $R_{\text {vir }}$. The subsample cuts, number of galaxies, median halo masses, and median redshifts for each subsample are listed in Table 2.

In the sample, rank correlation tests yield no statistically significant correlations between the galaxy redshift, azimuthal angle, inclination, and halo mass. A one-dimensional Kolmogorov-Smirnov (KS) test was also carried out on the galaxy orientation measurements to test whether the sample is unbiased. We find that the azimuthal angles of the galaxies are consistent with that of unbiased samples at the $0.6 \sigma$ level; the inclination angles of the galaxies are also consistent with that of unbiased samples at the $2.3 \sigma$ level.

\subsection{Quasar Spectra}

The details of the quasar spectra are found in Kacprzak et al. (2015) and Nielsen et al. (2017), but we summarize them here. Each of the 23 quasars has a medium resolution $\left(R \sim 20,000\right.$, FWHM $\left.\sim 18 \mathrm{~km} \mathrm{~s}^{-1}\right)$ spectrum from $H S T / C O S$. Voigt profiles were fitted to each of the OVI $\lambda \lambda 1031,1037$ doublet absorption lines with VPFIT (Carswell \& Webb 2014), and the zero-points of velocity (i.e., $z_{\text {abs }}$ ) were defined as the velocity where $50 \%$ of the modeled absorption resides on each side in the optical depth distribution for the O vi $\lambda 1031$ line (Nielsen et al. 2017). The velocity bounds of the absorption were defined to be where modeled absorption deviates from the continuum (value of 1 ) by $1 \%$ (to 0.99 ). The absorption profiles for each absorber-galaxy pair are plotted in Appendix A, where the velocity zero-point corresponds to the systemic velocity of the host galaxy.

\subsection{Pixel-Velocity Two-Point Correlation Function}

The TPCF method has previously been used to analyze the absorber velocity dispersions of $\mathrm{Mg}$ II and O VI absorbers surrounding galaxies (Nielsen et al. 2015, 2016, 2017, 2018; Pointon et al. 2017). In the first part of this work, we investigate the mass dependence of the velocity dispersions of the absorbers ("absorber kinematics"). For the rest of this work, we shift the velocities relative to the galaxy systemic velocity to investigate the motion of the surrounding gas relative to the galaxy ("absorber-galaxy kinematics").

\subsubsection{Absorber Kinematics}

The details of the absorber TPCF construction are expounded in Nielsen et al. (2016), but we briefly summarize the method here. The velocities of all the pixels, within the velocity bounds in which OVI absorption is formally detected, are first extracted for a desired subsample (e.g., all edge-on galaxies) and combined into a single array. Statistically, for a given subsample, this step makes the equivalency between a single quasar absorption sightline around multiple galaxies, and a single galaxy with multiple sightlines. We then calculate the absolute value of the velocity separations between every pair of pixel velocities in the subsample, $\Delta v_{\text {pix }}$. These velocity separations are binned into $20 \mathrm{~km} \mathrm{~s}^{-1}$ bins to account for the resolution of COS, and the number of counts in each bin is normalized by the total number of velocity separation pairs in the subsample. This yields a probability distribution function of the velocity dispersion, the absorber TPCF.

The uncertainties on the TPCFs were determined by a bootstrap analysis with 100 bootstrap realizations where absorbergalaxy pairs were randomly drawn with replacement. These are $1 \sigma$ uncertainties from the mean of the bootstrap realizations. To characterize the TPCFs, we use the quantities $\Delta v(50)$ and $\Delta v(90)$. These represent the velocity separations within which $50 \%$ and $90 \%$ of the area under the TPCF is located. The corresponding uncertainties for $\Delta v(50)$ and $\Delta v(90)$ were also determined from the bootstrap analysis, where we obtained the $1 \sigma$ uncertainties from the mean of the bootstrap realizations. Following Nielsen et al. (2015), we employ two-sample $\chi^{2}$ tests to examine and quantify statistical differences between the TPCFs of different galaxyabsorber subsamples. We report the reduced $\chi^{2}$, i.e., $\chi_{\nu}^{2}$, where $\nu$ is the number of degrees of freedom.

\subsubsection{Absorber-Galaxy Kinematics}

The TPCFs described in the previous section were modified to account for the velocity of the gas relative to the host galaxy. After the pixel velocities are extracted from a subsample, they are shifted with respect to the host galaxy,

$$
v_{\text {pix-gal }}=\left|v_{\text {pix }}+c \frac{z_{\text {abs }}-z_{\text {gal }}}{1+z_{\text {gal }}}\right| \text {. }
$$

We take the absolute value of this shifted velocity to quantify the velocity dispersion of the absorbing gas with respect 
to the galaxy systemic velocity, without considering its direction. We also do not know whether the gas is physically located in front of or behind the host galaxy, so we cannot determine if the gas is infalling or outflowing relative to the galaxy, hence the velocity sign is not important. Thus, in this work, the absorber-galaxy TPCF is defined to be a statistical measure of the velocity dispersion of the absorbers whose velocities are shifted with respect to the galaxy systemic velocity.

Since our sample of galaxies spans a range of halo masses, we account for the galaxy halo mass by normalizing the shifted velocities by the circular velocity at the observed impact parameter of the host galaxy, $V_{\mathrm{c}}(D)$. We now work with the circular velocity-normalized pixel-galaxy velocities, $v_{\text {pix }- \text { gal }} / V_{\mathrm{c}}(D)$. Once these values are obtained, the normalized velocities for a given subsample are combined and are subtracted between every possible pair of pixels. Thus we obtain normalized pixel-galaxy velocity separations, $\Delta\left(v_{\text {pix-gal }} / V_{\mathrm{c}}(D)\right)$.

For the absorber-galaxy TPCF, we use a bin size of $\Delta\left(v_{\text {pix-gal }} / V_{\mathrm{c}}(D)\right)=0.2$, which is determined by dividing the maximum uncertainty of the systemic galaxy redshift, $\Delta z_{\text {gal }}=0.0001$, corresponding to $\sim 30 /\left(1+\left\langle z_{\text {gal }}\right\rangle\right) \mathrm{km} \mathrm{s}^{-1}$ in the galaxy rest frame, by the average $V_{\mathrm{c}}(D)$ in the sample, $156 \mathrm{~km} \mathrm{~s}^{-1}$.

\subsection{Average Absorption Profiles}

We complement the TPCFs with the average absorption profiles for a given subsample. The average profiles provide supplementary information about the optical depth distribution and indicate how much gas there is at a given velocity.

The average absorption profiles were constructed by first extracting the pixel velocities from the individual OVI $\lambda 1031$ absorption profiles. For absorber kinematics, we do not modify these velocities. For absorber-galaxy kinematics, we shift the velocities relative to the galaxy systemic velocity and normalize them with respect to the galaxy's circular velocity, $V_{\mathrm{c}}(D)$. The associated flux values, obtained using model absorption profiles to the data to remove any contamination due to noise and blends on the flux, are also extracted. Next, for any given profile, the positive pixel velocities and negative pixel velocities are separated into two arrays. We take the absolute value of the negative velocities because we do not know where the gas is located relative to the galaxy other than the projected distance, thus the sign on the velocities bears little meaning. Then for both the velocity and flux arrays we run a linear interpolation routine onto a standardized velocity grid to place all absorbers on the same velocity or absorbergalaxy normalized velocity scale. This is repeated for all the absorber-galaxy pairs in the subsample.

The final average absorption profile is then obtained by calculating the average flux over all absorber-galaxy pairs in the subsample, for each velocity bin. The uncertainties on the absorption, like the TPCFs, were determined by a bootstrap analysis with 100 bootstrap realizations, where absorbergalaxy pairs were randomly drawn with replacement. These are $1 \sigma$ uncertainties from the mean of the bootstrap realizations.

The individual OVI absorption profiles, shifted relative to the galaxy systemic velocity, are plotted in Appendix A for direct comparison to the absorber-galaxy kinematics.

\section{ABSORBER KINEMATICS}

We first investigate the dependence of OVI absorber kinematics on halo mass in Figure 1. The isolated galaxy sample is sliced by $\log \left(M_{\mathrm{h}} / M_{\odot}\right)=11.7$, which was motivated by Oppenheimer et al. (2016) who used it to define sub- $L^{*}$ and $L^{*}$ galaxies, which we call "lower mass" and "higher mass" galaxies here, respectively. We also show the group galaxy sample from Pointon et al. (2017) to study a more complete mass range for better comparison with the simulations. The group sample was defined by the number of galaxies and not mass since halo masses derived by halo abundance matching may not be representative of the entire group halo. Oppenheimer et al. (2016) defined group halos as those with $\log \left(M_{\mathrm{h}} / M_{\odot}\right) \geq 12.3$, but here we assume a more conservative mass of $\log \left(\bar{M}_{\mathrm{h}} / M_{\odot}\right)>12$ in the TPCF studies. If we calculate a mass for each group member galaxy with halo abundance matching and sum these values, we can obtain a lower limit on the group mass. We only use these values in Figure 2. While these group masses span all three mass subsamples, the group environments may have different absorption characteristics due to interaction effects (Alonso et al. 2012; Fernández et al. 2015; Pointon et al. 2017) so we consider these to be a separate sample.

In Figure 1, higher mass galaxies have significantly larger velocity dispersions than lower mass galaxies $(7.7 \sigma)$ and group galaxies $(13.1 \sigma)$, while group galaxies have similar velocity dispersions to lower mass galaxies $(1.8 \sigma)$. From Table 2 , the $\Delta v(50)$ and $\Delta v(90)$ measurements for the lower mass galaxies $\left(80_{-8}^{+5} \mathrm{~km} \mathrm{~s}^{-1}\right.$ and $188_{-19}^{+11} \mathrm{~km} \mathrm{~s}^{-1}$, respectively) and group galaxies $\left(64_{-7}^{+9} \mathrm{~km} \mathrm{~s}^{-1}\right.$ and $153_{-18}^{+21} \mathrm{~km} \mathrm{~s}^{-1}$, respectively) are all consistent within uncertainties. However, higher mass galaxies have significantly larger $\Delta v(50)\left(108_{-9}^{+6} \mathrm{~km} \mathrm{~s}^{-1}\right)$ and $\Delta v(90)\left(255_{-22}^{+14} \mathrm{~km} \mathrm{~s}^{-1}\right)$ values than for either lower mass galaxies or group galaxies.

We tested the robustness of this trend to outliers in column density by redo-ing the TPCF analysis without the two absorber-galaxy pairs corresponding to $\log N_{\text {Ovi }}>15.0$. The significances change slightly to $5.7 \sigma, 12.2 \sigma$, and $1.8 \sigma$, respectively, but the inferences remain the same. Additionally, we tested whether the column density variation in the three different mass bins was the cause for the kinematic trends, where total column density and total velocity spread may be related. For this we normalized each pixel velocity by the total column density for the given absorber. While the results are not plotted here, we found that the significances did not change drastically, with $8.0 \sigma, 12 \sigma$, and $0.7 \sigma$, respectively. Thus the results we present in Figure 1 are kinematic trends rather than some underlying column density trend.

This effect is also observed in the average absorption profiles plotted in the bottom panel of Figure 1. Higher mass galaxies have the most optical depth at all line of sight velocities compared to lower mass and group galaxies, while group galaxies have the least optical depth at all line of sight velocities. We determined column densities for each average absorption profile by mirroring the plotted profile over $v_{\text {pixel }}=0 \mathrm{~km} \mathrm{~s}^{-1}$ (the resulting profile is symmetric) and modeling the profiles with VPFIT as was done for the actual O VI profiles. We find column densities of $\log N_{\mathrm{OVI}}=14.570$ for higher mass galaxies, $\log N_{\mathrm{OVI}}=14.44$ for lower mass galaxies, and $\log N_{\mathrm{OVI}}=14.18$ for group galaxies. As expected, the higher mass galaxies have the largest column densities and group galaxies have the smallest.

Because we are probing different mass galaxies with our 

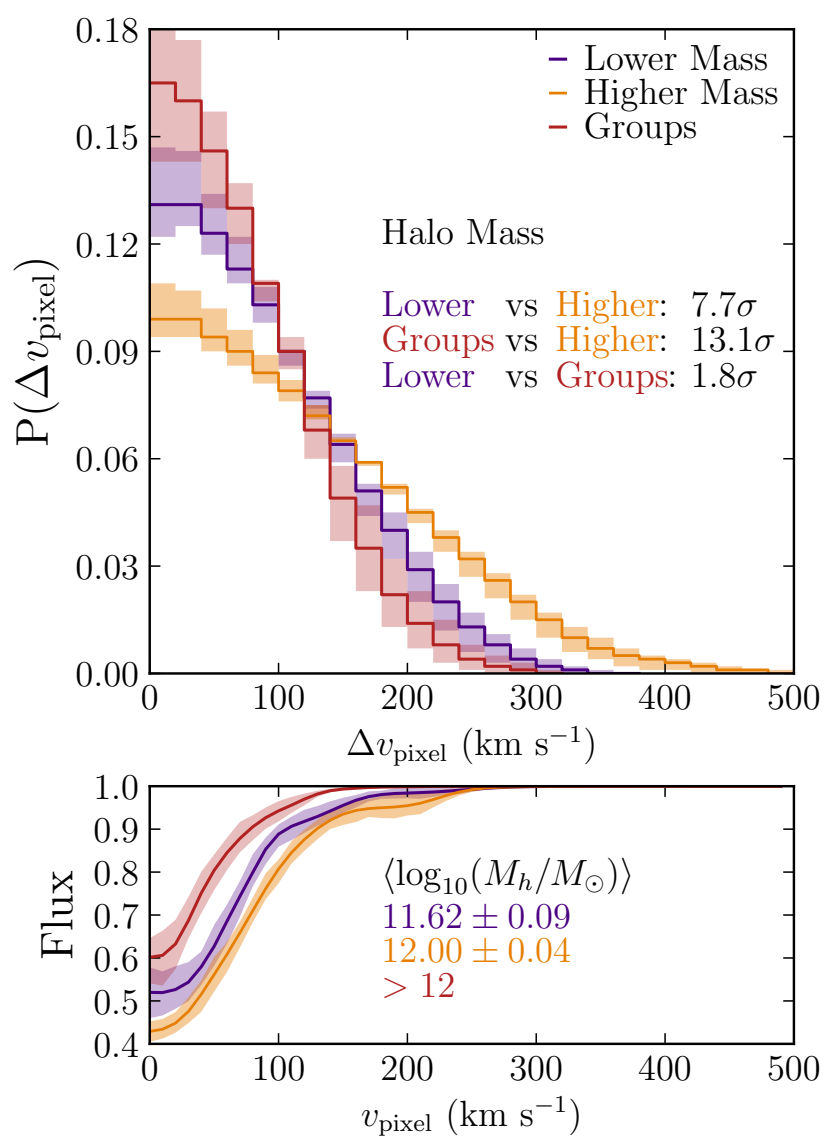

FIG. 1.- (Top) Absorber TPCFs for lower mass (purple), higher mass (orange), and group (red) galaxies. The higher mass galaxies have significantly larger velocity dispersions compared with the lower mass galaxies $(7.7 \sigma)$ and group galaxies $(13.1 \sigma)$. Group galaxies from Pointon et al. (2017) have similar velocity dispersions to lower mass galaxies. (Bottom) Average absorption profiles corresponding to lower mass galaxies, higher mass galaxies, and group galaxies. Group galaxies have lower optical depth at all pixel velocities compared with lower and higher mass galaxies. $1 \sigma$ uncertainties for both the TPCFs and average absorption spectra were calculated with a bootstrap analysis with 100 bootstrap realizations.

quasar sightlines, we may be biased toward probing the inner regions of more massive galaxies. This is important because OVI equivalent widths and column densities decrease with increasing impact parameter (e.g., Tumlinson et al. 2011; Kacprzak et al. 2015). The median impact parameter normalized by the virial radius, $D / R_{\text {vir }}$, for lower mass galaxies is 0.67 , for higher mass galaxies is 0.46 , and for group galaxies is 0.47 . However, a KS test comparing the $D / R_{\text {vir }}$ distributions suggest that the lower and higher mass galaxy subsamples were drawn from the same population $(2.1 \sigma)$. Comparing to the group galaxy sample, we find significances of $0.0 \sigma$ for group galaxies versus higher mass galaxies and $0.6 \sigma$ for group galaxies versus lower mass galaxies. The $D / R_{\text {vir }}$ ranges for all three subsamples are also consistent. Thus differences in $D / R_{\text {vir }}$ between the subsamples does not appear to strongly influence our results.

To better compare the kinematics results with the Oppenheimer et al. (2016) simulations, we plot our data and the simulation data on the column density-mass plane in Figure 2. The observational data plotted are line-of-sight column densities and halo masses from halo abundance matching. Halo masses of group galaxies are measured using the

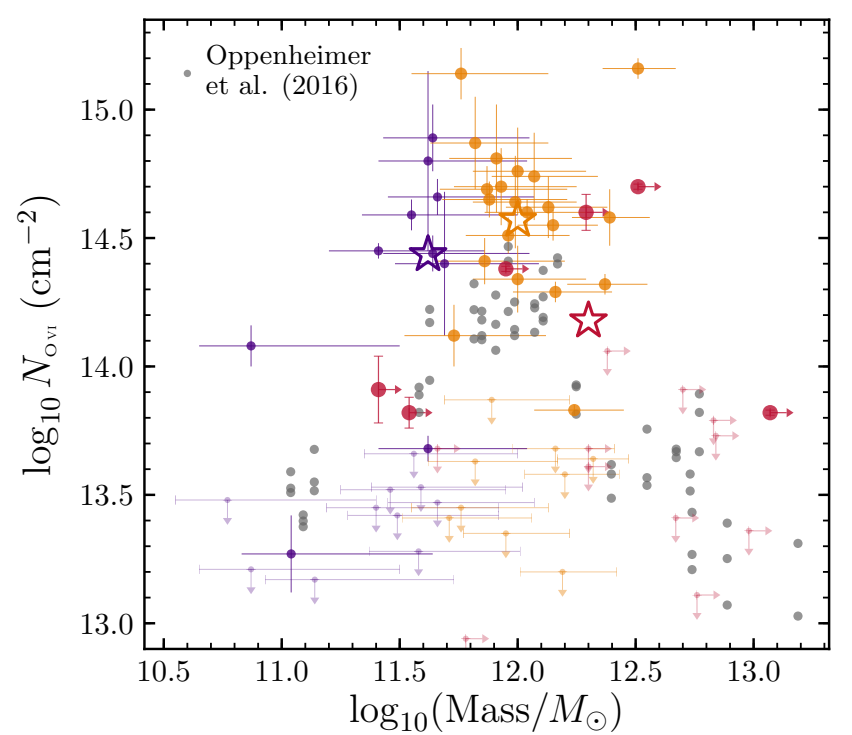

FIG. 2.- Column densities and masses for observational and simulation data. Lower mass galaxies are represented by purple points, higher mass are orange, and group galaxies are red. Because the group galaxy masses are difficult to estimate, their masses are plotted as lower limits. Upper limits on absorption are plotted as small points with downward arrows. Stars indicate the column densities obtained from the average absorption profiles presented in Figure 1, where the halo mass is determined by the median halo mass for the lower and higher mass subsamples. Gray points are the aperture column densities within $150 \mathrm{kpc}$ and $M_{200}$ masses from Oppenheimer et al. (2016). Our data follow the trend of increasing column densities toward a maximum for $L^{*}$ galaxies, and decreasing toward the highest masses.

same method, but the individual galaxy masses are summed for each group to give a lower limit. Points are colored and sized by the three halo mass bins from Figure 1. The simulation data are plotted as gray points and represent aperture column densities within $D=150 \mathrm{kpc}$ as a function of $\log \left(M_{200} / M_{\odot}\right)$. The column densities measured from the average absorption profiles in the previous paragraph are plotted as stars for each mass subsample. Our data generally follow the simulated trend that column densities increase with increasing mass up to $\log \left(M_{\mathrm{h}} / M_{\odot}\right) \sim 12.0$ and decrease with mass above. Note that upper limits on the OVI column densities from Kacprzak et al. (2015) and Pointon et al. (2017) are plotted for completeness, but they are not studied here since we focus on kinematics, which non-absorbers do not have by definition. The upper limits mostly lie below $\log N_{\text {OvI }}=13.7$ regardless of halo mass, indicating that the OVI CGM is inherently patchy. We leave further analysis to later work.

\section{ABSORBER-GALAXY KINEMATICS}

In this section we examine the relative absorber-galaxy kinematics using the TPCFs described in Section 2.3.2. Note that we normalize pixel-galaxy velocities by the circular velocity at the observed impact parameter, $V_{\mathrm{c}}(D)$, in order to assess the kinematics in a mass-independent way. This is done in light of the results of the previous section, which showed a significant mass-dependence in the absorber kinematics. We also only focus on isolated galaxy sightlines (i.e., no group environment kinematics from Figure 1) due to the velocity normalization. Since the OVI gas is in an intra-group medium and is not specifically associated with a particular galaxy, we cannot assign a single $V_{\mathrm{c}}(D)$ for each group absorber. 
TABLE 2

TPCF $\Delta v(50)$ AND $\Delta v(90)$ MEASUREMENTS

\begin{tabular}{|c|c|c|c|c|c|c|c|}
\hline Subsample & \# galaxies & Cut 1 & Cut 2 & $\left\langle\log \left(M_{\mathrm{h}} / M_{\odot}\right)\right\rangle$ & $\left\langle z_{\mathrm{gal}}\right\rangle$ & $\Delta v(50)$ & $\Delta v(90)$ \\
\hline & \multicolumn{5}{|c|}{ Figure 1: Absorber Kinematics } & \multicolumn{2}{|c|}{$v=v_{\text {pix }}\left(\mathrm{km} \mathrm{s}^{-1}\right)$} \\
\hline Lower Mass & 10 & $\log \left(M_{\mathrm{h}} / M_{\odot}\right)<11.7$ & $\ldots$ & $11.62 \pm 0.09$ & $0.21 \pm 0.03$ & $80_{-8}^{+5}$ & $188_{-19}^{+11}$ \\
\hline Higher Mass & 21 & $\log \left(M_{\mathrm{h}} / M_{\odot}\right) \geq 11.7$ & $\ldots$ & $12.00 \pm 0.04$ & $0.32 \pm 0.03$ & $108_{-9}^{+6}$ & $255_{-22}^{+19}$ \\
\hline \multirow[t]{2}{*}{ Group $^{a}$} & 6 & $\ldots$ & $\ldots$ & $\ldots$ & $0.19 \pm 0.05$ & $64_{-7}^{+9}$ & $153_{-18}^{+21}$ \\
\hline & \multicolumn{5}{|c|}{ Figure 3: Absorber-Galaxy Kinematics } & \multicolumn{2}{|c|}{$v=v_{\text {pix }-\mathrm{gal}} / V_{\mathrm{c}}(D)$} \\
\hline Lower Mass & 15 & $\log \left(M_{\mathrm{h}} / M_{\odot}\right)<11.88$ & $\ldots$ & $11.64 \pm 0.04$ & $0.22 \pm 0.03$ & $0.45_{-0.03}^{+0.03}$ & $1.12_{-0.09}^{+0.08}$ \\
\hline \multirow[t]{2}{*}{ Higher Mass } & 16 & $\log \left(M_{\mathrm{h}} / M_{\odot}\right) \geq 11.88$ & $\ldots$ & $12.06 \pm 0.07$ & $0.34 \pm 0.05$ & $0.34_{-0.02}^{+0.02}$ & $0.87_{-0.07}^{+0.06}$ \\
\hline & \multicolumn{5}{|c|}{ Figure 4: Absorber-Galaxy Kinematics } & \multicolumn{2}{|c|}{$v=v_{\text {pix }- \text { gal }} / V_{\mathrm{c}}(D)$} \\
\hline Lower-z & 15 & $z_{\mathrm{gal}}<0.244$ & $\ldots$ & $11.73 \pm 0.18$ & $0.205 \pm 0.016$ & $0.40_{-0.04}^{+0.04}$ & $1.11_{-0.12}^{+0.11}$ \\
\hline \multirow[t]{2}{*}{ Higher-z } & 16 & $z_{\mathrm{gal}} \geq 0.244$ & $\ldots$ & $11.95 \pm 0.05$ & $0.39 \pm 0.03$ & $0.37_{-0.02}^{+0.02}$ & $0.92_{-0.05}^{+0.04}$ \\
\hline & \multicolumn{5}{|c|}{ Not Plotted: Absorber-Galaxy Kinematics } & \multicolumn{2}{|c|}{$v=v_{\text {pix }-\mathrm{gal}} / V_{\mathrm{c}}(D)$} \\
\hline Face-on & 15 & $i<51^{\circ}$ & $\ldots$ & $11.82 \pm 0.14$ & $0.32 \pm 0.09$ & $0.40_{-0.04}^{+0.03}$ & $1.03_{-0.12}^{+0.09}$ \\
\hline Edge-on & 16 & $i \geq 51^{\circ}$ & $\ldots$ & $11.91 \pm 0.08$ & $0.24 \pm 0.05$ & $0.38_{-0.03}^{+0.02}$ & $0.96_{-0.08}^{+0.07}$ \\
\hline Major Axis & 17 & $\Phi<45^{\circ}$ & $\ldots$ & $11.76 \pm 0.16$ & $0.23 \pm 0.06$ & $0.35_{-0.03}^{+0.02}$ & $0.90_{-0.09}^{+0.06}$ \\
\hline \multirow[t]{2}{*}{ Minor Axis } & 14 & $\Phi \geq 45^{\circ}$ & $\ldots$ & $11.92 \pm 0.06$ & $0.33 \pm 0.05$ & $0.42_{-0.03}^{+0.04}$ & $1.07_{-0.10}^{+0.10}$ \\
\hline & \multicolumn{5}{|c|}{ Figure 5: Absorber-Galaxy Kinematics } & \multicolumn{2}{|c|}{$v=v_{\text {pix }- \text { gal }} / V_{\mathrm{c}}(D)$} \\
\hline Major Axis + Face-on & 10 & $\Phi<45^{\circ}$ & $i<51^{\circ}$ & $12.00 \pm 0.12$ & $0.32 \pm 0.05$ & $0.35_{-0.03}^{+0.02}$ & $0.87_{-0.09}^{+0.06}$ \\
\hline Major Axis + Edge-on & 7 & $\Phi<45^{\circ}$ & $i \geq 51^{\circ}$ & $11.69 \pm 0.32$ & $0.26 \pm 0.07$ & $0.38_{-0.05}^{+0.06}$ & $0.97_{-0.17}^{+0.20}$ \\
\hline Minor Axis + Face-on & 5 & $\Phi \geq 45^{\circ}$ & $i<51^{\circ}$ & $11.64 \pm 0.17$ & $0.18 \pm 0.05$ & $0.51_{-0.06}^{+0.04}$ & $1.24_{-0.16}^{+0.11}$ \\
\hline \multirow[t]{2}{*}{ Minor Axis + Edge-on } & 9 & $\Phi \geq 45^{\circ}$ & $i \geq 51^{\circ}$ & $11.96 \pm 0.13$ & $0.32 \pm 0.08$ & $0.38_{-0.03}^{+0.00}$ & $0.96_{-0.08}^{+0.07}$ \\
\hline & \multicolumn{5}{|c|}{ Figure 6: Absorber-Galaxy Kinematics } & \multicolumn{2}{|c|}{$v=v_{\mathrm{pix}-\mathrm{gal}} / V_{\mathrm{c}}(D)$} \\
\hline Major Axis + Lower Mass & 7 & $\Phi<45^{\circ}$ & $\log \left(M_{\mathrm{h}} / M_{\odot}\right)<11.88$ & $11.66 \pm 0.15$ & $0.31 \pm 0.09$ & $0.40_{-0.04}^{+0.04}$ & $1.02_{-0.16}^{+0.15}$ \\
\hline Major Axis + Higher Mass & 10 & $\Phi<45^{\circ}$ & $\log \left(M_{\mathrm{h}} / M_{\odot}\right) \geq 11.88$ & $12.09 \pm 0.09$ & $0.29 \pm 0.06$ & $0.33_{-0.03}^{+0.02}$ & $0.83_{-0.09}^{+0.06}$ \\
\hline Minor Axis + Lower Mass & 8 & $\Phi \geq 45^{\circ}$ & $\log \left(M_{\mathrm{h}} / M_{\odot}\right)<11.88$ & $11.64 \pm 0.05$ & $0.20 \pm 0.02$ & $0.48_{-0.04}^{+0.03}$ & $1.17_{-0.11}^{+0.09}$ \\
\hline Minor Axis + Higher Mass & 6 & $\Phi \geq 45^{\circ}$ & $\log \left(M_{\mathrm{h}} / M_{\odot}\right) \geq 11.88$ & $12.04 \pm 0.14$ & $0.41 \pm 0.07$ & $0.37_{-0.03}^{+0.03}$ & $0.92_{-0.11}^{+0.10}$ \\
\hline Face-on + Lower Mass & 6 & $i<51^{\circ}$ & $\log \left(M_{\mathrm{h}} / M_{\odot}\right)<11.88$ & $11.65 \pm 0.09$ & $0.22 \pm 0.08$ & $0.51_{-0.05}^{+0.03}$ & $1.23_{-0.14}^{+0.08}$ \\
\hline Face-on + Higher Mass & 9 & $i<51^{\circ}$ & $\log \left(M_{\mathrm{h}} / M_{\odot}\right) \geq 11.88$ & $12.00 \pm 0.15$ & $0.30 \pm 0.06$ & $0.33_{-0.02}^{+0.03}$ & $0.81_{-0.06}^{+0.08}$ \\
\hline Edge-on + Lower Mass & 9 & $i \geq 51^{\circ}$ & $\log \left(M_{\mathrm{h}} / M_{\odot}\right)<11.88$ & $11.64 \pm 0.10$ & $0.23 \pm 0.04$ & $0.40_{-0.03}^{+0.03}$ & $0.99_{-0.10}^{+0.11}$ \\
\hline Edge-on + Higher Mass & 7 & $i \geq 51^{\circ}$ & $\log \left(M_{\mathrm{h}} / M_{\odot}\right) \geq 11.88$ & $12.13 \pm 0.08$ & $0.39 \pm 0.08$ & $0.37_{-0.03}^{+0.03}$ & $0.93_{-0.10}^{+0.09}$ \\
\hline
\end{tabular}

a Data from Pointon et al. (2017).

\subsection{Bivariate Analysis}

We again slice the sample by mass, but choose the median halo mass of the sample $\left(\left\langle\log \left(M_{\mathrm{h}} / M_{\odot}\right)\right\rangle=11.88\right)$ in order to have roughly equal numbers of galaxies in each mass bin for a later multivariate analysis. The absorber-galaxy TPCFs and associated average absorption profiles for lower mass and higher mass galaxies are plotted in Figure 3. After accounting for the inherent mass bias in the absorber kinematics above, we find that lower mass galaxies $\left(\log \left(M_{\mathrm{h}} / M_{\odot}\right)<11.88\right)$ have larger absorber-galaxy velocity dispersions compared with higher mass galaxies $\left(\log \left(M_{\mathrm{h}} / M_{\odot}\right) \geq 11.88\right)$ at a significance of $4.5 \sigma$. From Table $2, \Delta v(50)$ and $\Delta v(90)$ for lower mass galaxies $\left(0.45_{-0.03}^{+0.03}\right.$ and $1.12_{-0.09}^{+0.08}$, respectively) are larger than that for higher mass galaxies $\left(0.34_{-0.02}^{+0.02}\right.$ and $0.87_{-0.07}^{+0.06}$, respectively).

The average absorption profiles below the TPCFs in Figure 3 show that the bulk of the absorption lies around the galaxy systemic velocity, and this result applies to all subsequent subsample slices. Furthermore, most of the absorption lies within the host galaxy's circular velocity at the observed 

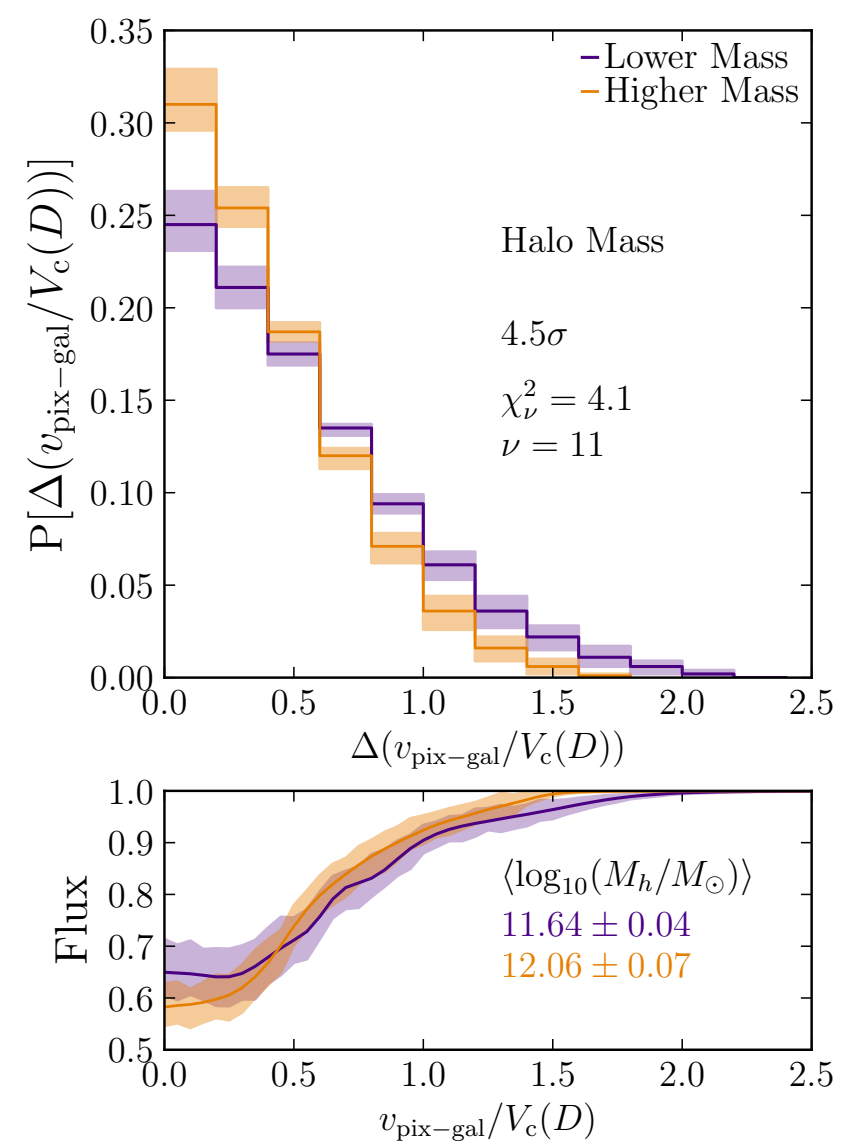

FIG. 3.- (Top) Normalized absorber-galaxy TPCFs for lower mass and higher mass galaxies. Unlike Figure 1, the pixel-galaxy velocities are normalized by the circular velocity at the observed impact parameter, $V_{\mathrm{c}}(D)$, to account for the range of halo masses in the sample. This shows a strong mass-dependence for the kinematics associated with OVI at the $4.5 \sigma$ level, where lower mass galaxies have significantly larger velocity dispersions than higher mass galaxies. (Bottom) The average absorption spectra corresponding to lower mass galaxies and higher mass galaxies. $1 \sigma$ uncertainties for both the TPCFs and average absorption spectra were calculated with a bootstrap analysis with 100 bootstrap realizations.

impact parameter, indicating that this gas is likely bound to the galaxy. In a complement to the TPCFs, the average absorption profile for lower mass galaxies extends to larger normalized pixel-galaxy velocities than for higher mass galaxies. This small fraction of the absorption profiles has velocities greater than $V_{c}(D)$, indicating that this gas may not be bound to the host galaxies, and the fraction may be larger for lower mass galaxies.

To account for any redshift evolution of the absorbergalaxy kinematics, we cut the sample by the median redshift, $\left\langle z_{\mathrm{gal}}\right\rangle=0.244$. This was motivated by work done in Nielsen et al. (2016), who found that the Mg II absorber velocity dispersions for red galaxies decreased with decreasing redshift, possibly indicative of the quenching of star formation. In Figure 4 , there is a weak suggestion that lower redshift galaxies $\left(\left\langle z_{\text {gal }}\right\rangle<0.244\right)$ have larger absorber-galaxy velocity dispersions than higher redshift galaxies $\left(\left\langle z_{\text {gal }}\right\rangle \geq 0.244\right)$ at a significance of $2.6 \sigma$. This result trends in the opposite direction as that found with $\mathrm{MgII}$, although here we are investigating the relative absorber-galaxy velocity dispersions rather than absorber velocity dispersions. Referring to Table 2, $\Delta v(50)$ measurements for lower redshift galaxies and higher redshift
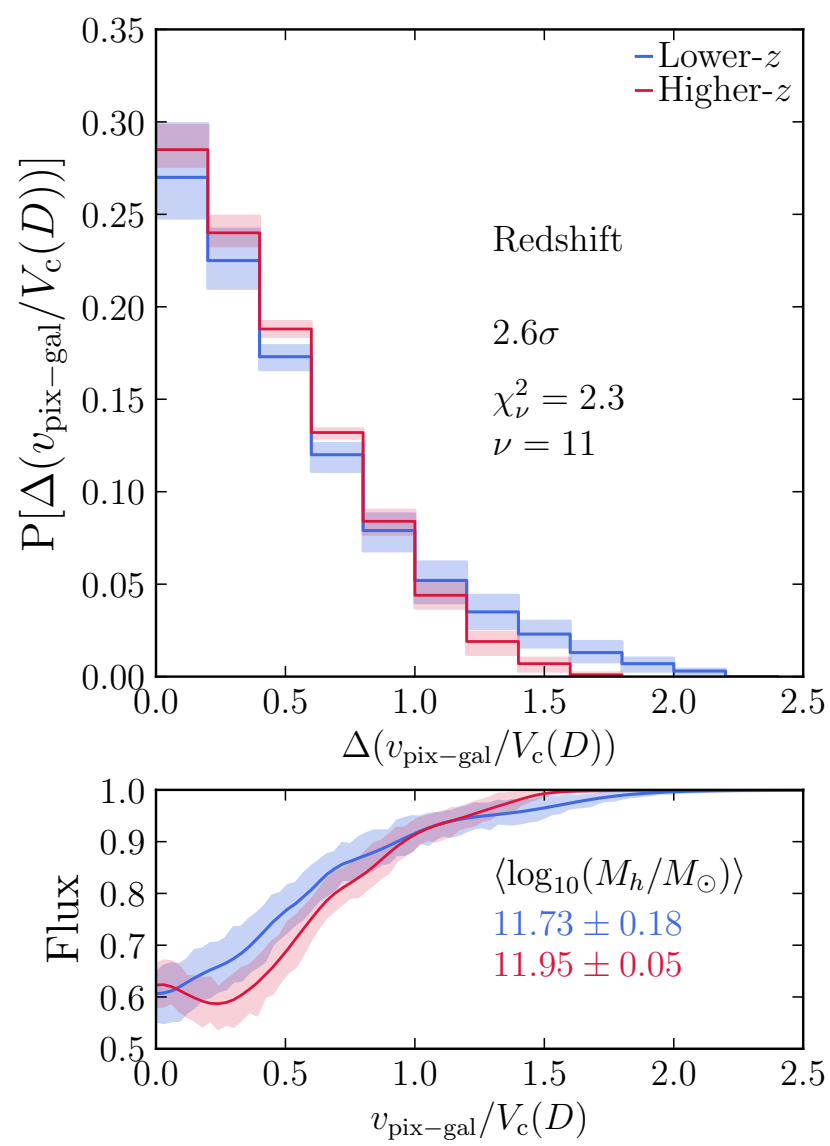

FIG. 4.- (Top) Normalized absorber-galaxy TPCFs for lower redshift and higher redshift galaxies. There is a probable redshift dependence of the OVI kinematics at the $2.6 \sigma$ level, where OVI absorbing gas in lower redshift galaxies has larger velocities relative to $\mathrm{O}$ VI absorbing gas in higher redshift galaxies. (Bottom) The average absorption spectra corresponding to lower and higher redshift galaxies. $1 \sigma$ uncertainties for both the TPCFs and the average spectra were calculated with a bootstrap analysis with 100 bootstrap realizations.

galaxies are similar, although $\Delta v(90)$ for lower redshift galaxies is slightly larger than for higher redshift galaxies. The median masses for the two subsamples are consistent within uncertainties, so any differences seen here are likely not dominated by the mass dependence of Figure 3. The average absorption profiles in the bottom panel of Figure 4 show that lower redshift galaxies have a nontrivial optical depth at a larger normalized pixel-galaxy velocity compared with the higher redshift galaxies, but the two profiles are still consistent within uncertainties. It is possible that OVI kinematics have a redshift dependence, analogous to that of Mg II kinematics in red galaxies (Nielsen et al. 2016), but this investigation would be better done on a sample with a greater range in redshifts (up to $z \sim 2-3$ ).

We also cut the sample by the median inclination, $\langle i\rangle=51^{\circ}$, where galaxies with $i \geq 51^{\circ}$ are considered "edge-on" galaxies and galaxies with $i<51^{\circ}$ are considered "face-on" galaxies. We find that both edge-on and face-on galaxies have the same velocity dispersions $(0.01 \sigma$, not plotted) and average absorption profiles. The $\Delta v(50)$ and $\Delta v(90)$ measurements reported in Table 2 are consistent within uncertainties. The median masses and median redshifts for the two subsamples are consistent within uncertainties.

A final bivariate cut made to the sample is the median az- 

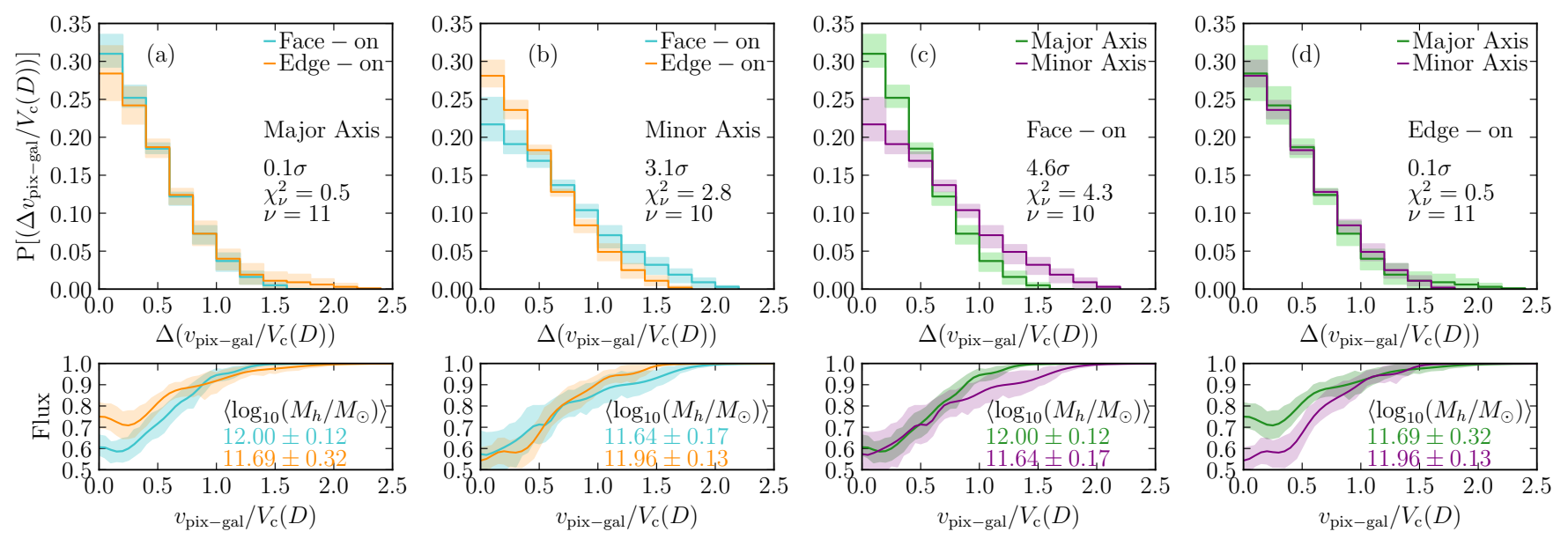

FIG. 5.- (Top) Normalized absorber-galaxy TPCFs for a) edge-on and face-on galaxies probed along the major axis; b) edge-on and face-on galaxies probed along the minor axis; c) face-on galaxies probed along the major and minor axes; d) edge-on galaxies probed along the major and minor axes. (Bottom) Average absorption profiles corresponding to the top panels. In a) and b), cyan corresponds to face-on galaxies, and orange corresponds to edge-on galaxies. In c) and d), green corresponds to galaxies probed along the major axis, and purple corresponds to galaxies probed along the minor axis. The uncertainties for the average absorption profiles were calculated with a bootstrap analysis with 100 bootstrap realizations.

imuthal angle of $\langle\Phi\rangle=45^{\circ}$, where galaxies with $\Phi \geq 45^{\circ}$ are considered "minor axis" galaxies, that is, galaxies that are probed along their projected minor axis. "Major axis" galaxies, where $\Phi<45^{\circ}$, are defined in a similar fashion. We find that minor axis galaxies have similar velocity dispersions to major axis galaxies ( $1.5 \sigma$, not plotted), and the average absorption profiles are not qualitatively different. From Table 2, the $\Delta v(50)$ and $\Delta v(90)$ measurements for both major axis galaxies and minor axis galaxies are inconsistent within uncertainties, but are still within $1.4 \sigma$. Additionally, the median masses and median redshifts for the major axis galaxies and minor axis galaxies are consistent within uncertainties.

The independence of the OVI kinematics on the inclination and azimuthal angles reported here has been confirmed in previous work (Nielsen et al. 2017; Kacprzak et al. 2019), but here we have obtained the same conclusion with absorbergalaxy kinematics for simple bivariate cuts.

\subsection{Multivariate Analysis}

\subsubsection{Inclination and Azimuthal Angle}

In Figure 5, we present the TPCFs for galaxy subsamples cut by inclination, $i$, as well as galaxy azimuthal angle, $\Phi$, for all halo masses. The corresponding average absorption profiles are plotted in the panels below the TPCFs.

In Figure 5(a), we compare face-on (cyan lines) and edgeon (orange lines) galaxies probed along their projected major axis. There is no difference in the absorber-galaxy velocity dispersions between the two inclinations $(0.1 \sigma)$. The associated $\Delta v(50)$ and $\Delta v(90)$ measurements presented in Table 2 are consistent within uncertainties, providing further support that the velocity dispersions between face-on and edge-on inclinations for major axis galaxies are very similar. The median masses for the two subsamples are consistent within uncertainties. The average absorption profile shows that there is a slight tail for the edge-on major axis galaxies at higher normalized pixel-galaxy velocities. While this is reflected in the TPCFs, the difference between the plotted subsamples is not significant due to large uncertainties. There is also more optical depth near the galaxy systemic velocity for the faceon major axis galaxies compared with the edge-on major axis galaxies.
Figure 5(b) compares face-on (cyan lines) and edge-on (orange lines) inclinations as well, but for galaxies probed along their projected minor axis. There is a significant difference in the velocity dispersions between the two inclinations $(3.1 \sigma)$, where face-on galaxies probed along the minor axis have a slightly larger velocity dispersion than edge-on galaxies. From Table 2, the associated $\Delta v(50)$ measurements for face-on minor axis galaxies $\left(0.51_{-0.06}^{+0.04}\right)$ are larger than for edge-on minor axis galaxies $\left(0.38_{-0.03}^{+0.02}\right)$. Additionally, $\Delta v(90)$ for face-on minor axis galaxies $\left(1.24_{-0.16}^{+0.10}\right)$ is larger than for edge-on minor axis galaxies $\left(0.96_{-0.08}^{+0.07}\right)$. This may be attributable to the observation that edge-on minor axis galaxies $\left(\left\langle\log \left(M_{\mathrm{h}} / M_{\odot}\right)\right\rangle=11.96 \pm 0.13\right)$ tend to be of higher mass than that of face-on minor axis galaxies $\left(\left\langle\log \left(M_{\mathrm{h}} / M_{\odot}\right)\right\rangle=\right.$ $11.64 \pm 0.17)$, so the corresponding halos have a larger virial temperature. This provides the conditions for OVI to ionize into higher order species and thus reduces the size and kinematic extent of the OVI clouds (Oppenheimer et al. 2016; Pointon et al. 2017). The average absorption profiles clearly show that there is a larger optical depth at higher normalized velocities for the face-on minor axis galaxies compared with the edge-on minor axis galaxies. This is seen as the tail in the TPCF, but the uncertainties in the TPCFs are large. The average absorption profile shows no differences in the absorption between the two subsamples around the galaxy systemic velocity.

Figure 5(c) compares face-on galaxies probed along the major axis (green lines) and minor axis (purple lines). There is a very significant difference $(4.6 \sigma)$ in the velocity dispersions, with minor axis galaxies showing a larger velocity dispersion compared with major axis galaxies in face-on $\left(i<51^{\circ}\right)$ inclinations. This is also seen in the $\Delta v(50)$ and $\Delta v(90)$ measurements reported in Table 2, where $\Delta v(50)$ for face-on galaxies probed along the minor axis $\left(0.51_{-0.06}^{+0.04}\right)$ is significantly larger than when probed along the major axis $\left(0.35_{-0.03}^{+0.02}\right)$. Likewise, the $\Delta v(90)$ value for face-on galaxies probed along the minor axis $\left(1.24_{-0.16}^{+0.10}\right)$ is significantly larger than face-on galaxies probed along the major axis $\left(0.87_{-0.09}^{+0.06}\right)$. The median mass for face-on minor axis galaxies, $\left\langle\log \left(M_{\mathrm{h}} / M_{\odot}\right)\right\rangle=$ $11.64 \pm 0.17$, is lower than that for face-on major axis galax- 
ies, $\left\langle\log \left(M_{\mathrm{h}} / M_{\odot}\right)\right\rangle=12.00 \pm 0.12$, where the direction of the difference reflects the result of Figure 3. The mass difference likely plays a role in the significant difference in the TPCFs, rather than being only an inclination effect. The average absorption profile also shows a much larger optical depth at higher normalized velocities (largely for $v_{\text {pix }- \text { gal }} / V_{\mathrm{c}}(D)>1$, where the gas is more likely to be unbound) for face-on minor axis galaxies compared with the face-on major axis galaxies, which is seen as the tail in the TPCF. Where the gas is expected to be bound, there is no difference in the average absorption profiles around the galaxy systemic velocity.

Finally, Figure 5(d) compares edge-on galaxies probed along the major axis (green lines) and minor axis (purple lines). There is no difference $(0.1 \sigma)$ in the velocity dispersions between the two azimuthal angle categories. This is reflected in the overlap of the respective $\Delta v(50)$ and $\Delta v(90)$ measurements reported in Table 2 . The median masses for edge-on major axis galaxies and edge-on minor axis galaxies are consistent within uncertainties. The average absorption profiles also have similar optical depths at the larger normalized velocities, but there is a larger optical depth for the edgeon minor axis galaxies around the galaxy systemic velocity compared with the edge-on major axis galaxies.

\subsubsection{Halo Masses and Orientation}

Given the significant difference in the absorber-galaxy kinematics for lower mass galaxies compared to higher mass galaxies, the results in the previous section with galaxy inclinations and azimuthal angles may largely be due to the distribution of galaxy masses in each subsample comparison. In Figure 6, we present TPCFs for galaxy subsamples cut by halo mass, $\log \left(M_{\mathrm{h}} / M_{\odot}\right)$, and one of two galaxy orientation measures: inclination, $i$, or azimuthal angle, $\Phi$. The corresponding average absorption profiles are plotted in the panels below the TPCFs. We conduct this test to better pinpoint the galaxy properties that are most important in governing the absorbergalaxy kinematics.

Figure 6(a) compares lower mass (indigo lines) and higher mass (orange lines) galaxies probed along the major axis. There is no difference $(1.0 \sigma)$ in the velocity dispersions, though the lower mass galaxies have a slightly wider velocity dispersion tail (but with large uncertainties) than higher mass galaxies when probed along the major axis. This is reflected in the consistency, within uncertainties, of the $\Delta v(50)$ and $\Delta v(90)$ measurements from Table 2 between the two subsamples. The average absorption profiles are also consistent within uncertainties.

Figure 6(b) shows the TPCFs for minor axis galaxies. There is a significant difference $(3.1 \sigma)$ between the velocity dispersions of the lower mass (indigo lines) and higher mass (orange lines) galaxies, where lower mass galaxies have a larger velocity dispersion than higher mass galaxies probed along the minor axis. The difference in velocity dispersions is also seen in the $\Delta v(50)$ and $\Delta v(90)$ measurements reported in Table 2 , where these values for lower mass galaxies $\left(0.48_{-0.04}^{+0.03}\right.$ and $1.17_{-0.11}^{+0.07}$, respectively) are larger than for higher mass galaxies $\left(0.37_{-0.03}^{+0.03}\right.$ and $0.92_{-0.11}^{+0.10}$, respectively). While this difference was expected from Figure 3 , the fact that the comparison for galaxies probed along the projected major axis in Figure 6(a) is insignificant indicates that azimuthal angle plays a role in the observed kinematic structure. However, the average absorption profiles for the two subsamples are comparable within uncertainties.
In Figure 6(c), we compare lower and higher mass TPCFs for face-on galaxies. There is a very significant difference $(6.7 \sigma)$ between the velocity dispersions of the lower mass (indigo lines) and higher mass (orange lines) galaxies, where lower mass face-on galaxies have a much larger velocity dispersion than higher mass face-on galaxies. The difference in velocity dispersions is reflected in the $\Delta v(50)$ and $\Delta v(90)$ measurements from Table 2, where the values for the lower mass face-on galaxies $\left(0.51_{-0.05}^{+0.03}\right.$ and $1.23_{-0.14}^{+0.08}$, respectively) are much greater than for higher mass, face-on galaxies $\left(0.33_{-0.02}^{+0.03}\right.$ and $\left.0.81_{-0.06}^{+0.08}\right)$. The average absorption profiles also show that the optical depth at higher normalized velocities is much larger for the lower mass face-on galaxies compared with the higher mass face-on galaxies.

In Figure 6(d), we compare the TPCFs of lower mass and higher mass galaxies with edge-on inclinations. There is no difference $(0.3 \sigma)$ between the velocity dispersions of the lower mass edge-on (indigo lines) and higher mass edge-on (orange lines) galaxies. This is seen in the consistency between their $\Delta v(50)$ and $\Delta v(90)$ measurements. The massdependence found in Figure 3 is not present for edge-on galaxies, but is for face-on galaxies in the previous paragraph, suggesting that galaxy inclination is important for determining the absorber-galaxy kinematics. The average absorption profiles of the lower mass edge-on galaxies and the higher mass edge-on galaxies are very similar, where the bulk of the absorption occurs around the galaxy systemic velocity, and the optical depth tapers off at similar normalized velocities.

Finally, we tested similar mass bins for the orientation subsamples, although these comparisons are not plotted directly. There are no significant differences between major and minor axis galaxies for either the lower mass $(0.8 \sigma)$ or higher mass $(0.4 \sigma)$ subsamples. Similarly, there is no significant difference between edge-on and face-on galaxies for the higher mass subsample. However, lower mass face-on galaxies have significantly larger velocity dispersions compared to edge-on lower mass galaxies $(3.3 \sigma)$. In this section, the clear outlier in kinematics is thus the lower mass face-on subsample.

\section{DISCUSSION}

From the TPCFs and average absorption profiles presented above, we find that there is a strong mass dependence of the O VI absorber kinematics, where higher mass $\left(\log \left(M_{\mathrm{h}} / M_{\odot}\right) \geq\right.$ 11.7) isolated galaxies have larger velocity dispersions compared with lower mass $\left(\log \left(M_{\mathrm{h}} / M_{\odot}\right)<11.7\right)$ isolated galaxies. Group galaxies have much narrower velocity dispersions than the higher mass isolated galaxies $(13.1 \sigma)$. We also find that the absorber-galaxy kinematics display non-virialized motions. These are primarily due to outflows in face-on and minor axis orientations for lower mass galaxies. These motions were found after normalizing the pixel-galaxy velocities by the circular velocity at the observed impact parameter, $V_{\mathrm{c}}(D)$, to account for the range of halo masses in the sample.

\subsection{Absorber Kinematics}

The mass dependence of the TPCFs and average absorption profiles in Figure 1 may be attributable to the strength of the OVI absorption, quantified by the column density, $\log N_{\text {OvI }}$. Simulations of OVI in galaxy halos show that the column density reaches its apex at $\log \left(M_{\mathrm{h}} / M_{\odot}\right)=12$ because the virial temperature of these galaxies is comparable to the temperature at which the OVI ionization fraction is greatest (Oppenheimer et al. 2016; also see Nelson et al. 2018 and Oppenheimer et al. 2018 for an alternative explanation). Galaxy 

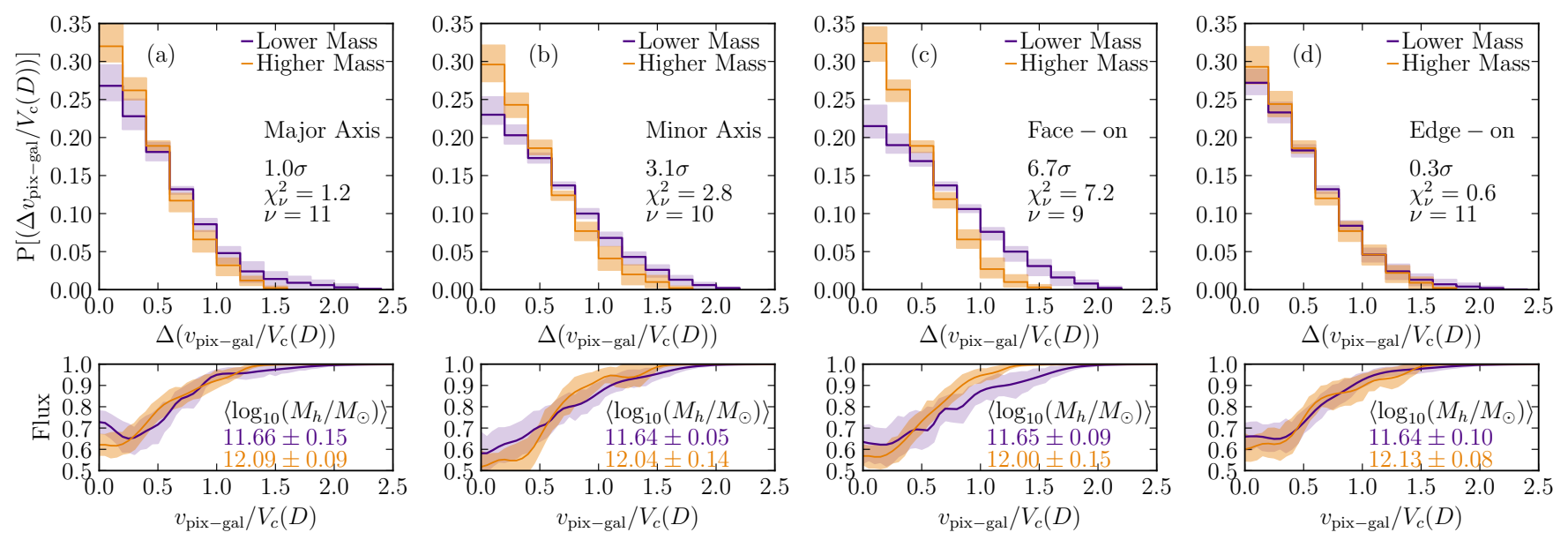

FIG. 6.- (Top) Normalized absorber-galaxy TPCFs for lower mass and higher mass galaxies probed along a) the major axis; b) the minor axis, and seen in c) face-on inclinations; d) edge-on inclinations. (Bottom) Average absorption profiles corresponding to the top panels. Orange corresponds to higher mass galaxies, and purple corresponds to lower mass galaxies. The uncertainties were calculated with a bootstrap analysis with 100 bootstrap realizations.

halos with lower $\left(\log \left(M_{\mathrm{h}} / M_{\odot}\right)<11.7\right)$ or higher masses $\left(\log \left(M_{\mathrm{h}} / M_{\odot}\right)>12.3\right.$, i.e., group environments) have lower column densities or weaker OVI absorption. This is likely because a smaller (larger) galaxy halo mass corresponds to a halo that is too cold (hot) for a significant fraction of OVI. We confirm this trend in the observational column densities plotted in Figure 2. This relation may also lead to smaller O VI clouds and a statistically lower kinematic extent for sub- $L^{*}$ and group galaxies.

We clearly see this effect in the absorber kinematics. The median mass for higher mass galaxies corresponds to $\sim L^{*}$ galaxies, which have a virial temperature that is most conducive for the presence of OVI gas according to Oppenheimer et al. (2016). Our results indicate that this also leads to a larger kinematic extent of the OVI gas since there is more gas to be distributed along the line of sight. In contrast, lower mass galaxies at sub- $L^{*}$ likely have virial temperatures which are too cool for a significant presence of $\mathrm{OVI}$, resulting in more narrow kinematic dispersions. As shown by Pointon et al. (2017) and confirmed here, group galaxies are the other extreme in that they likely live in halos with virial temperatures that are too warm for OVI. As OVI ionizes out into higher order species (e.g., OVII and beyond) for these highest mass galaxies, this translates to narrower kinematic extents because there is less gas along the line of sight at the temperatures required for strong OVI absorption.

We also saw this mass-OVI effect in the optical depth distribution of the average absorption profiles, constructed from the OVI $\lambda 1031$ transition. For all pixel velocities, higher mass galaxies $\left(\log \left(M_{\mathrm{h}} / M_{\odot}\right) \approx 12\right)$ have more absorption compared to both lower mass galaxies $\left(\log \left(M_{\mathrm{h}} / M_{\odot}\right)<11.7\right)$ and group environments $\left(\log \left(M_{\mathrm{h}} / M_{\odot}\right) \gtrsim 12.3\right)$. As shown in Figure 2, the column densities obtained from these average absorption profiles, $\log N_{\mathrm{OvI}}=14.44,14.57$, and 14.18 for increasing halo mass, follow a similar trend with mass as the aperture column densities obtained by Oppenheimer et al. (2016) in the EAGLE simulations. ${ }^{2}$

Previous absorber kinematics work by Nielsen et al. (2017) did not investigate this mass dependence, rather they focused

\footnotetext{
2 There is a well-known tension between observations and simulations, where simulations under-produce OVI absorption (e.g., Oppenheimer et al. 2016; Suresh et al. 2017), and this is the cause of the offset between the observed and simulated points.
}

on the star formation properties of the galaxies with $B-K$ colors. They found that the kinematics did not depend on galaxy orientations or star formation activity and suggested that the observed gas may be a result of ancient outflows which have had time to form a roughly kinematically uniform halo. They also suggested that differing ionization conditions throughout the CGM result in the azimuthal angle dependence of OVI found by Kacprzak et al. (2015). Combined with the results presented here, we suggest that the absorption properties of $\mathrm{OVI}$ are not good straightforward probes of current baryon cycle processes in galaxies. Instead, O VI absorption properties are primarily probes of halo mass.

\subsection{Absorber-Galaxy Kinematics}

In an attempt to better pull out baryon cycle signatures in the OVI absorption profiles, we shifted the absorption relative to the galaxy systemic velocity and normalized these pixel-galaxy velocities by the galaxy circular velocity at the observed impact parameter, $V_{c}(D)$. The resulting absorbergalaxy kinematics are effectively independent of the trends found with the absorber kinematics. Thus the differences between subsamples for the absorber-galaxy kinematics should not be dominated by mass and, due to studying the relative velocities between the observed gas and the host galaxies, may reflect baryon cycle processes.

We first tested this by examining the relative absorbergalaxy kinematics for lower and higher mass galaxies in Figure 3. Overall, the bulk of the absorption lies near the galaxy systemic velocity, with little gas exceeding the galaxy circular velocity, similar to previous work comparing to the galaxy escape velocity (Tumlinson et al. 2011; Mathes et al. 2014; Kacprzak et al. 2019). The small fraction of gas that does exceed $V_{c}(D)$ is more likely to escape the galaxy, and this fraction is slightly larger for lower mass galaxies, which have statistically larger $(4.5 \sigma)$ absorber-galaxy kinematics. Mathes et al. (2014) examined a sample of 11 galaxies with measured OVI absorption and found that the gas around lower mass galaxies is more likely to escape the halo than higher mass galaxies. The authors suggested this is evidence for differential kinematics due to differential wind recycling, where outflowing gas in higher mass galaxies is more likely to recycle back onto the host galaxy due to lower outflow velocities relative to $V_{c}(D)$ (Oppenheimer et al. 2010). 
Mathes et al. (2014) also presented CGM radial velocities for a simulated $\log \left(M_{\mathrm{h}} / M_{\odot}\right) \simeq 11.3$ galaxy. Radially infalling gas rarely exceeded $V_{c}(D)$ beyond $40 \mathrm{kpc}$ due to the velocities being determined by the galaxy's potential well, where the gas is largely subject to gravitational forces inward and potentially (weaker) forces that slow down the material as it travels toward the galaxy. Conversely, outflowing gas often exceeded $V_{c}(D)$ out to at least $140 \mathrm{kpc}$. The differences in the TPCF kinematics we present here are in the regime where the gas flows exceed $V_{c}(D)$ at large impact parameters. Thus we rule out accretion as the dominant source of this material and further suggest that much of the OVI traces outflowing gas.

We examined this further by studying the kinematics as a function of galaxy orientation, where outflows are expected to dominate sightlines probing galaxies along their projected minor axis or probing face-on galaxies. Simple bivariate cuts with galaxy inclination or azimuthal angle did not result in significant differences between the kinematics. This is likely due to other factors such as comparable halo masses between the subsamples where differential kinematics would not be obvious. Additionally, outflows are not expected along the projected major axis of edge-on galaxies, or for major axis galaxies with inclinations closer to being face-on. Thus, we sliced the sample further.

Accretion/rotation line-of-sight velocities are maximized for edge-on galaxies along the major axis (at least for low impact parameter, e.g., Steidel et al. 2002; Kacprzak et al. 2010; Stewart et al. 2011; Danovich et al. 2012, 2015), whereas outflow velocities are minimized (e.g., Rubin et al. 2014). Examining these orientations, we found no significant difference in the absorber-galaxy TPCFs between face-on galaxies and edge-on galaxies probed along their projected major axes $(0.1 \sigma)$, as shown in Figure 5(a). The TPCFs presented in Figure 5(d), comparing major and minor axis edge-on galaxies, are also consistent $(0.1 \sigma)$. The only differences for these subsamples are in the average absorption profiles near the galaxy systemic velocity, where larger optical depths are found for the face-on galaxies or along the minor axis. These latter differences are not due to a dependence on halo mass since the subsamples in both comparisons have similar median halo masses and the mass dependence is normalized out. Instead, these results may be a signature of ancient outflows, which OVI may trace (e.g., Ford et al. 2014). In an edge-on inclination, outflows manifest as minor axis absorption centered around the galaxy systemic velocity since the radial velocity component is mostly perpendicular to a galaxy's disk. We see this signature as more absorption along the minor axis, and less along the major axis where accreting gas is preferred.

A potential problem for interpreting OVI gas origins is introduced via the quasar sightline technique. Recently, Kacprzak et al. (2019) examined the relative velocities between OVI and the host galaxy rotation curves for absorbers along the projected major axis. They found that observed $\mathrm{O}$ VI does not correlate with galaxy rotation and has velocity dispersions that span the entire range of galaxy rotation velocities. In contrast, the authors found inflowing O VI filaments in the CGM of simulated galaxies. The discrepancy between the observed and simulated data is a result of the observational technique, where the observed kinematic signatures of gas infall/rotation are blurred due to multiple kinematic structures along the lines-of-sight which cannot be disentangled with the data currently available (also see Churchill et al. 2015; Peeples et al. 2018). Additionally, OVI absorbing clouds are predicted to be large, with radii on the order of tens to hun- dreds of kiloparsecs from photoionization modeling (Lopez et al. 2007; Muzahid 2014; Hussain et al. 2015; Stern et al. 2016). ${ }^{3}$ Since accreting filaments tend to have small covering fractions (e.g., Martin et al. 2012; Rubin et al. 2012), gas infall signatures are overwhelmed by outflow signatures.

We now focus on orientations in which outflows are expected to dominate kinematic signatures: face-on galaxies and galaxies probed along their projected minor axes. In Figure 5(b), there is a significant difference between faceon and edge-on galaxies probed along the minor axis $(3.1 \sigma)$, where the velocity dispersions are larger for face-on galaxies. The difference likely comes about because outflow velocities are minimized in edge-on inclinations but are maximized for face-on inclinations. The average absorption profiles are similar within uncertainties, but there is a suggestion that the edgeon subsample is more centrally concentrated near the galaxy systemic velocity, as expected for a bipolar outflow geometry. The optical depth profile is more smooth for the face-on subsample out to large normalized pixel velocities, likely reflecting varying outflowing velocities with impact parameter. This is supported by the results of Kacprzak et al. (2019), who found that minor axis OVI gas can be modeled by a decelerating outflow.

In another comparison, Figure 5(c), we found that faceon galaxies probed along the projected minor axis have significantly larger velocity dispersions than face-on major axis galaxies $(4.6 \sigma) .{ }^{4}$ One might expect outflows to dominate the signature in all face-on galaxies, so this result seems unusual. However, the face-on minor axis subsample has a larger impact parameter on average, $\langle D\rangle=95.7 \mathrm{kpc}$, than the faceon major axis subsample, $\langle D\rangle=75.6 \mathrm{kpc}$. We expect that outflows should influence the observed gas more strongly at lower impact parameter (e.g., Bordoloi et al. 2011), which is opposite the result we find. Since the major axis subsample has significantly smaller velocity dispersions at lower impact parameters, outflows do not appear to dominate the face-on major axis absorption signature as strongly as along the minor axis. Furthermore, the major axis subsample has a larger median mass than the minor axis subsample. In the differential kinematics/recycling scenario, less massive galaxies can host larger $V_{\mathrm{c}}(D)$ normalized velocities due to smaller potential wells, which is what we observe. The combination of large absorber-galaxy velocity dispersions and large impact parameters along the minor axis of face-on galaxies in Figure 5(c) is interesting given the simulation results presented in Kacprzak et al. (2019). The authors found that for simulated galaxies at $z=1$ with $\log \left(M_{\mathrm{h}} / M_{\odot}\right) \sim 11.7$, minor axis OVI outflows accelerate out to $D \sim 50 \mathrm{kpc}$, where the gas begins to decelerate and later falls back onto the host galaxy. Perhaps the larger mean impact parameter for our face-on minor axis sample reflects the build-up of OVI gas due to this velocity turn-over, making the gas more easily observed.

The ease at which outflowing gas signatures are observed in lower mass face-on galaxies over other subsamples in Figure 6 could be explained with the differential kinematics/wind recycling described by Oppenheimer et al. (2010) and Mathes et al. (2014). The specific star formation rate is expected to be larger for lower mass galaxies, where lower mass galaxies

\footnotetext{
${ }^{3}$ Though note that the Oppenheimer et al. (2016) results assume OVI is collisionally ionized. It is more likely that $\mathrm{OVI}$ is some combination of photoionized and collisionally ionized.

${ }^{4}$ Recall that "face-on" here means $i<51^{\circ}$, which is quite inclined from a strict definition of "face-on", so the azimuthal angles are well-modeled in these systems.
} 
are more likely to be actively creating their stellar populations from recent star formation (e.g., Feulner et al. 2005). In this instance, outflowing gas in lower mass galaxies is likely more recent, which reduces the amount of time available for OVI gas to kinematically mix within the CGM (evidence for this kinematic mixing of OVI is discussed in Nielsen et al. 2017 and "kinematic blurring" arguments due to many lineof-sight structures are detailed in Kacprzak et al. 2019). Thus, the kinematic signatures of outflows along the line-of-sight are more likely to be preserved for lower mass galaxies than higher mass galaxies.

Unlike the absorber kinematics, the absorber-galaxy kinematics indirectly depend on halo mass. If mass was the most important galaxy property governing the kinematics even after normalizing by $V_{\mathrm{c}}(D)$, then every panel in Figure 6 would show significant differences between lower and higher mass subsamples. However, the only significant differences found are for comparisons in which outflows are expected to dominate the kinematics of at least one of the subsamples. Overall, the lower mass face-on galaxy subsample is the outlier, with velocity dispersions that are significantly larger than both higher mass face-on galaxies $(6.7 \sigma)$ and lower mass edge-on galaxies $(3.3 \sigma)$. This is consistent with the differential kinematics/wind recycling scenario (Oppenheimer et al. 2010; Mathes et al. 2014).

\section{SUMMARY AND CONCLUSIONS}

We first examined the absorber kinematics for OVI with the TPCF method employed in Nielsen et al. (2015, 2016, 2017, 2018); Pointon et al. (2017). The rest of the work analyzed absorber-galaxy kinematics for OVI gas using a modified version of the TPCFs. We used a subset of 31 galaxies from the "Multiphase Galaxy Halos" Survey (Kacprzak et al. 2015, 2019; Muzahid et al. 2015, 2016; Nielsen et al. 2017; Pointon et al. 2017, 2019) for the TPCF analysis; these are isolated galaxies, where they have no neighboring galaxies within $200 \mathrm{kpc}$ and within a line-of-sight velocity separation of $500 \mathrm{~km} \mathrm{~s}^{-1}$. The galaxies span $0.12<z_{\mathrm{gal}}<0.66$, where $\sigma_{z}$ is better than $\sim 30 \mathrm{~km} \mathrm{~s}^{-1}$ (e.g., Kacprzak et al. 2019) and are found within a projected distance of $200 \mathrm{kpc}$ from the quasar sightline. The galaxies were imaged with ACS, WFC3 or WFPC2 on HST, and the associated morphological properties were modeled using GIM2D (Simard et al. 2002; Kacprzak et al. 2015).

We shifted the pixel velocities from the absorption spectra relative to the galaxy systemic velocities, and normalized them with respect to the circular velocity at the impact parameter, $V_{\mathrm{c}}(D)$, to account for the range of halo masses $\left(10.9 \leq \log \left(M_{\mathrm{h}} / M_{\odot}\right) \leq 12.5\right)$ in the sample. We also found that this was crucial to eliminate any potential mass biases in the results. We analyzed absorber-galaxy kinematics of the subsamples derived from cuts by the halo mass, $\log \left(M_{\mathrm{h}} / M_{\odot}\right)$, galaxy redshift, $z_{\mathrm{gal}}$, azimuthal angle, $\Phi$, and inclination, $i$. The TPCFs are supplemented by average absorption profiles, which provide optical depth distribution information. We also show the individual absorption profiles in the Appendix.

We thus find that:

1. There is a mass dependence in the OVI absorber kinematics consistent with the column density-mass trends of Oppenheimer et al. (2016). Lower mass galaxies $\left(\log \left(M_{\mathrm{h}} / M_{\odot}\right)<11.7\right)$ tend to have narrower velocity dispersions than the typically more massive galaxies $\left(\log \left(M_{\mathrm{h}} / M_{\odot}\right) \sim 12\right)$. Subsamples with halo masses consistent with $L^{*}$ galaxies tend to have the largest velocity dispersions and this is reflected in both the TPCFs and the average absorption profiles. This mass subsample coincides with that of maximum OVI column density due to having a virial temperature comparable to the temperature at which the OVI ionization fraction is greatest. The largest halo masses, the group environment sample from Pointon et al. (2017), have the smallest velocity dispersions. This is likely due to more massive halos having a larger virial temperature, which provides the environment for OVI to be promoted to higher order species (e.g., OVII and beyond). The most massive halos are too hot for large OVI ionization fractions, thus reducing the size of OVI clouds and statistically leading to narrower kinematic extents.

2. The total OVI column densities for the lower mass, higher mass, and group samples are consistent with the aperture column densities from Oppenheimer et al. (2016), for $\log \left(M_{\mathrm{h}} / M_{\odot}\right) \geq 10.9$. This result provides further observational evidence of the virial temperature dependence of OVI.

3. After normalizing absorber-galaxy TPCFs by the circular velocity at the observed impact parameter, $V_{\mathrm{c}}(D)$, to account for halo mass, we found that lower mass galaxies $\left(\left\langle\log \left(M_{\mathrm{h}} / M_{\odot}\right)\right\rangle=11.64\right)$ have significantly larger (4.5 $\sigma$ ) absorber-galaxy velocity dispersions compared to higher mass galaxies $\left(\left\langle\log \left(M_{\mathrm{h}} / M_{\odot}\right)\right\rangle=12.06\right)$. The average absorption profiles demonstrate that there is a larger fraction of gas with velocities greater than $V_{\mathrm{c}}(D)$ for the lower mass subsample, suggestive of outflowing gas (e.g., Oppenheimer et al. 2010; Mathes et al. 2014; Kacprzak et al. 2019).

4. There are no significant differences for bivariate comparisons with subsamples sliced only by galaxy redshift $(2.6 \sigma)$, inclination $(0.01 \sigma)$, or azimuthal angle $(1.5 \sigma)$. However, these simple cuts neglect the fact that, e.g., gas along the projected major and minor axes of edgeon galaxies is expected to exhibit different kinematic signatures (e.g., Steidel et al. 2002; Kacprzak et al. 2010; Stewart et al. 2011; Bouché et al. 2013).

5. A multivariate analysis investigating subsamples sliced by galaxy inclination angle and azimuthal angle shows potential outflow signatures. Large velocity dispersions and optical depths beyond $V_{\mathrm{c}}(D)$ are found for orientations in which outflows are expected to be most optimal. Face-on minor axis subsamples have the largest kinematic dispersions and a smoothly decreasing optical depth distribution out to large normalized velocities, reflecting outflowing gas that is likely decelerating and will eventually return to the galaxy. Edge-on minor axis gas has large optical depths that are concentrated near the galaxy systemic velocity, suggesting a large quantity of OVI-absorbing gas being ejected perpendicular to the galaxy disk as expected for bipolar outflows. Accreting gas signatures are not directly observed for any subsample due to a combination of low covering fraction (e.g., Martin et al. 2012; Rubin et al. 2012) and "kinematic blurring" along the line-of-sight in which multiple kinematic structures are probed (e.g., Churchill et al. 2015; Peeples et al. 2018; Kacprzak et al. 2019).

6. Combining the mass and galaxy orientation subsamples, we found that while outflows are most easily 
observed in lower mass galaxies, the clear outlying subsample is lower mass face-on galaxies. In this case, more recent star formation in the lower mass galaxies than would be expected in higher mass galaxies results in the OVI gas having less time to mix kinematically to obscure the expected kinematic signatures of outflows.

With these results, we suggest that in order to understand the physics of OVI-absorbing gas, it is imperative to first consider the halo mass of the host galaxy. Combining the results of Nielsen et al. (2017) and those presented in Section 3, OVI absorber kinematics largely represent the underlying virial temperature of the host halo rather than baryon cycle processes, unlike lower ions such as Mg II (Nielsen et al. 2015, 2016). Furthermore, accounting for the Kacprzak et al. (2019) results for a subset of galaxies presented here, studying the circular velocity-normalized absorber-galaxy kinematics of OVI shows indications of outflow signatures, but the kinematic blurring due to multiple structures along the line-ofsight and the large cloud sizes predicted for OVI from photoionization models rule out easily detecting accreting gas. Due to this, it is becoming increasingly important for simulations to accurately model the CGM in order to better understand the origins of the observed gas, and therefore better understand how galaxies cycle their gas.

We thank the anonymous referee, whose comments improved the clarity of the manuscript. M.N. acknowledges the support from the Vacation Scholarship program at the Centre for Astrophysics \& Supercomputing at Swinburne Uni- versity of Technology. N.M.N. and G.G.K. acknowledge the support of the Australian Research Council through a Discovery Project DP170103470. S.K.P. acknowledges support through the Australian Government Research Training Program Scholarship. Parts of this research were supported by the Australian Research Council Centre of Excellence for All Sky Astrophysics in 3 Dimensions (ASTRO 3D), through project number CE170100012. S.M. acknowledges support from European Research Council (ERC), Grant Agreement 278594-GasAroundGalaxies. C.W.C. and J.C.C. acknowledge the support from award 1517831 from the National Science Foundation. J.C.C. is also supported by National Science Foundation grant AST-1312686. This work made use of Python packages NumPy and SciPy (Oliphant 2007), Matplotlib (Hunter 2007), IPython (Pérez \& Granger 2007), tqdm (da Costa-Luis et al. 2018), Numba (Lam et al. 2015), and of NASA's Astrophysics Data System Bibliographic Services.

Galaxy data presented here were obtained at the W. M. Keck Observatory, which is operated as a scientific partnership among the California Institute of Technology, the University of California, and the National Aeronautics and Space Administration. The Observatory was made possible by the generous financial support of the W. M. Keck Foundation. Observations were supported by Swinburne Keck programs 2014A_W178E, 2014B_W018E, 2015_W018E and 2016A_W056E. The authors wish to recognize and acknowledge the very significant cultural role and reverence that the summit of Mauna Kea has always had within the indigenous Hawaiian community. We are most fortunate to have the opportunity to conduct observations from this mountain.

Facilities: Keck:II (ESI)

\section{APPENDIX}

\section{A. ABSORPTION PROFILES FOR ABSORBER-GALAXY KINEMATICS}

Figure A1 presents the OVI absorption profiles for each absorber relative to the galaxy systemic redshift. These are the profiles that are used for the absorber-galaxy kinematics analyses presented in Section 4 (for the absorbers used in the absorber kinematics analysis in Section 3, see Nielsen et al. 2017). Absorbers are ordered by their host galaxy halo masses, with the field names, redshifts, and halo masses labeled above each panel pair. These panel pairs show both OVI lines, with $\lambda 1031$ plotted on top and $\lambda 1037$ on bottom. Data and uncertainties are plotted as black and green histograms, respectively. Voigt profile models are plotted as red curves while the individual fitted components are plotted as red ticks.

\section{REFERENCES}

Alonso, S., Mesa, V., Padilla, N., \& Lambas, D. G. 2012, A\&A, 539, A46

Bielby, R. M., Stott, J. P., Cullen, F., et al. 2019, MNRAS, 486, 21

Bordoloi, R., Lilly, S. J., Knobel, C., et al. 2011, ApJ, 743, 10

Bordoloi, R., Lilly, S. J., Hardmeier, E., et al. 2014, ApJ, 794, 130

Bouché, N., Hohensee, W., Vargas, R., et al. 2012, MNRAS, 426, 801

Bouché, N., Murphy, M. T., Kacprzak, G. G., et al. 2013, Science, 341, 50

Bryan, G. L., \& Norman, M. L. 1998, ApJ, 495, 80

Butler, M. J., Tan, J. C., Teyssier, R., et al. 2017, ApJ, 841, 82

Carswell, R. F., \& Webb, J. K. 2014, VPFIT: Voigt profile fitting program, Astrophysics Source Code Library

Chen, H.-W., Lanzetta, K. M., \& Webb, J. K. 2001, ApJ, 556, 158

Churchill, C. W., Mellon, R. R., Charlton, J. C., et al. 2000, ApJS, 130, 91 Churchill, C. W., Trujillo-Gomez, S., Nielsen, N. M., \& Kacprzak, G. G. 2013, ApJ, 779, 87

Churchill, C. W., Vander Vliet, J. R., Trujillo-Gomez, S., Kacprzak, G. G., \& Klypin, A. 2015, ApJ, 802, 10

Cole, S., Lacey, C. G., Baugh, C. M., \& Frenk, C. S. 2000, MNRAS, 319, 168

Cooper, T. J., Simcoe, R. A., Cooksey, K. L., O’Meara, J. M., \& Torrey, P. 2015, ApJ, 812, 58

Correa, C. A., Schaye, J., van de Voort, F., Duffy, A. R., \& Wyithe, J. S. B. 2018, MNRAS

Cox, T. J., Jonsson, P., Somerville, R. S., Primack, J. R., \& Dekel, A. 2008, MNRAS, 384, 386

da Costa-Luis, C., L., S., Mary, H., et al. 2018, tqdm/tqdm: tqdm v4.23.4 stable

Danovich, M., Dekel, A., Hahn, O., Ceverino, D., \& Primack, J. 2015 , MNRAS, 449, 2087
Danovich, M., Dekel, A., Hahn, O., \& Teyssier, R. 2012, MNRAS, 422, 1732

Fernández, M., Yuan, F., Shen, S., et al. 2015, Galaxies, 3, 156

Feulner, G., Gabasch, A., Salvato, M., et al. 2005, ApJ, 633, L9

Ford, A. B., Davé, R., Oppenheimer, B. D., et al. 2014, MNRAS, 444, 1260

Glidden, A., Cooper, T. J., Cooksey, K. L., Simcoe, R. A., \& O’Meara, J. M. 2016, ApJ, 833, 270

Gómez-Guijarro, C., Toft, S., Karim, A., et al. 2018, ApJ, 856, 121

Guillemin, P., \& Bergeron, J. 1997, A\&A, 328, 499

Ho, S. H., Martin, C. L., Kacprzak, G. G., \& Churchill, C. W. 2017, ApJ, 835,267

Hunter, J. D. 2007, Computing In Science \& Engineering, 9, 90

Hussain, T., Muzahid, S., Narayanan, A., et al. 2015, MNRAS S, 446, 2444

Johnson, S. D., Chen, H.-W., \& Mulchaey, J. S. 2013, MNRAS, 434, 1765

-. 2015, MNRAS, 449, 3263

Kacprzak, G. G. 2017, in Astrophysics and Space Science Library, Vol. 430, Gas Accretion onto Galaxies, ed. A. Fox \& R. Davé, 145

Kacprzak, G. G., Churchill, C. W., Barton, E. J., \& Cooke, J. 2011, ApJ, 733,105

Kacprzak, G. G., Churchill, C. W., Ceverino, D., et al. 2010, ApJ, 711, 533

Kacprzak, G. G., Churchill, C. W., Steidel, C. C., Spitler, L. R., \&

Holtzman, J. A. 2012, MNRAS, 427, 3029

Kacprzak, G. G., Muzahid, S., Churchill, C. W., Nielsen, N. M., \& Charlton, J. C. 2015, ApJ, 815, 22

Kacprzak, G. G., Vander Vliet, J. R., Nielsen, N. M., et al. 2019, ApJ, 870, 137

Kaviraj, S. 2014, MNRAS, 440, 2944

Klypin, A. A., Trujillo-Gomez, S., \& Primack, J. 2011, ApJ, 740, 102 

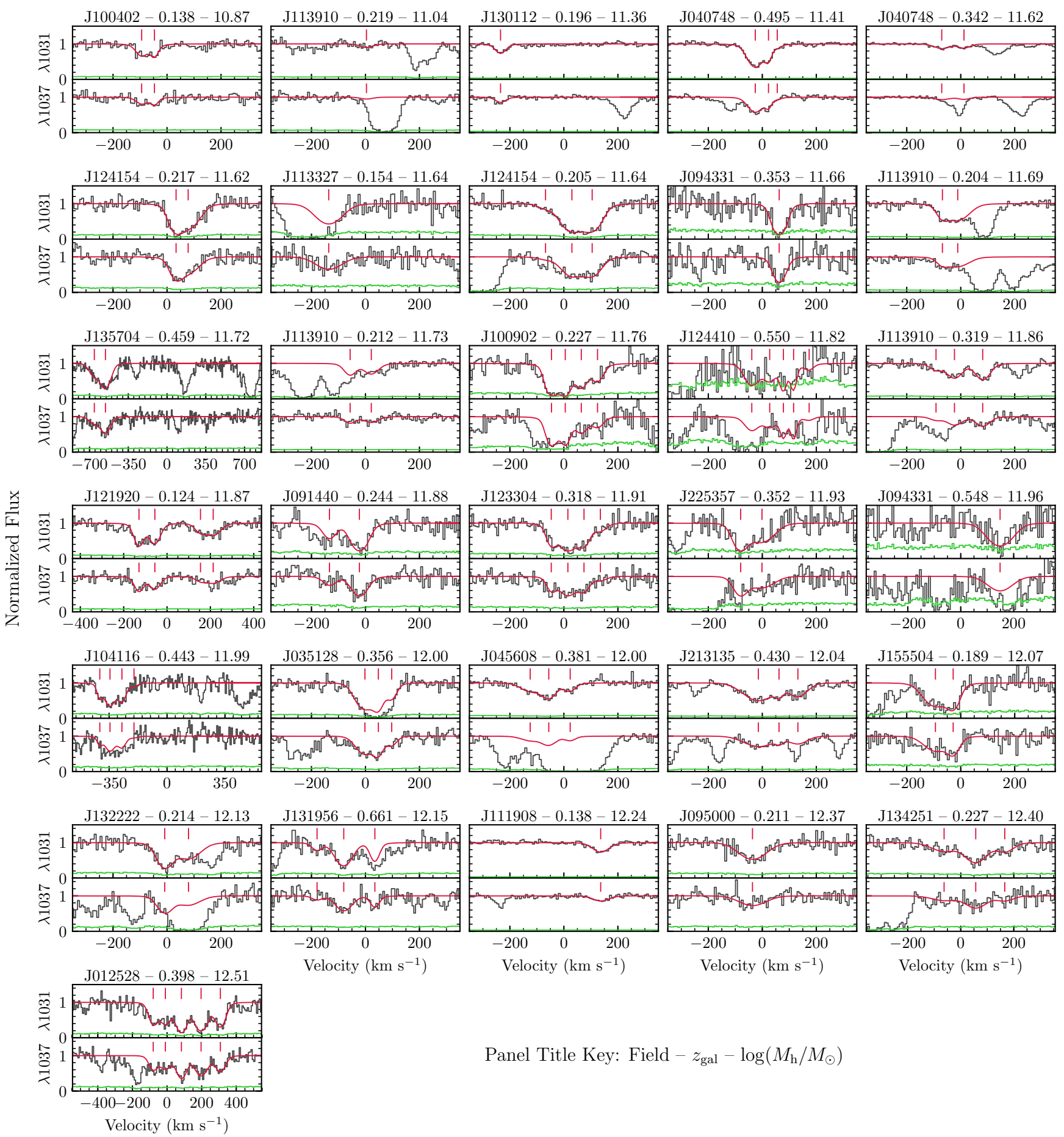

Panel Title Key: Field $-z_{\text {gal }}-\log \left(M_{\mathrm{h}} / M_{\odot}\right)$

FIG. A1.- Absorption profiles (black histogram) of the OVI $\lambda \lambda 1031,1037$ doublet along with the corresponding uncertainties (green line), and fits (red line), for each sample absorber-galaxy pair. The pixel velocities in the absorption spectra have been shifted relative to the galaxy systemic velocity. The original absorption spectra can be found in Nielsen et al. (2017). The OVI $\lambda 1031$ and OVI $\lambda 1037$ lines are plotted on the top and bottom, in each pair of panels, respectively. The vertical red ticks at the top of each panel represent the central velocity of each Voigt profile component fitted to the data. Note that the limits on the velocity axes are not all identical. 
Lam, S. K., Pitrou, A., \& Seibert, S. 2015, in Proceedings of the Second Workshop on the LLVM Compiler Infrastructure in HPC, LLVM '15 (New York, NY, USA: ACM), 7:1-7:6

Lambas, D. G., Alonso, S., Mesa, V., \& O’Mill, A. L. 2012a, A\&A, 539, A45

-. 2012b, A\&A, 539, A45

Lehner, N., Howk, J. C., Tripp, T. M., et al. 2013, ApJ, 770, 138

Lopez, S., Ellison, S., D’Odorico, S., \& Kim, T.-S. 2007, A\&A, 469, 61

Martin, C. L., Shapley, A. E., Coil, A. L., et al. 2012, ApJ, 760, 127

Mathes, N. L., Churchill, C. W., Kacprzak, G. G., et al. 2014, ApJ, 792, 128

Muzahid, S. 2014, ApJ, 784, 5

Muzahid, S., Kacprzak, G. G., Charlton, J. C., \& Churchill, C. W. 2016, ApJ, 823, 66

Muzahid, S., Kacprzak, G. G., Churchill, C. W., et al. 2015, ApJ, 811, 132

Navarro, J. F., Frenk, C. S., \& White, S. D. M. 1996, ApJ, 462, 563

Nelson, D., Kauffmann, G., Pillepich, A., et al. 2018, MNRAS, 477, 450

Nielsen, N. M., Churchill, C. W., Kacprzak, G. G., Murphy, M. T., \& Evans, J. L. 2015, ApJ, 812, 83

-. 2016, ApJ, 818, 171

Nielsen, N. M., Kacprzak, G. G., Muzahid, S., et al. 2017, ApJ, 834, 148

Nielsen, N. M., Kacprzak, G. G., Pointon, S. K., Churchill, C. W., \& Murphy, M. T. 2018, ArXiv e-prints, arXiv:1808.09562

Oliphant, T. E. 2007, Computing in Science \& Engineering, 9, 10

Oppenheimer, B. D., Davé, R., Kereš, D., et al. 2010, MNRAS, 406, 2325

Oppenheimer, B. D., Segers, M., Schaye, J., Richings, A. J., \& Crain, R. A. 2018, MNRAS, 474, 4740

Oppenheimer, B. D., Crain, R. A., Schaye, J., et al. 2016, MNRAS, 460, 2157

Ownsworth, J. R., Conselice, C. J., Mortlock, A., et al. 2014, MNRAS, 445, 2198

Peeples, M. S., Corlies, L., Tumlinson, J., et al. 2018, ArXiv e-prints, arXiv: 1810.06566

Pérez, F., \& Granger, B. E. 2007, Computing in Science and Engineering, 9, 21

Pointon, S. K., Kacprzak, G. G., Nielsen, N. M., et al. 2019, ApJ, submitted
Pointon, S. K., Nielsen, N. M., Kacprzak, G. G., et al. 2017, ApJ, 844, 23 Prochaska, J. X., Weiner, B., Chen, H.-W., Mulchaey, J., \& Cooksey, K. 2011, ApJ, 740, 91

Putman, M. E., Peek, J. E. G., \& Joung, M. R. 2012, ARA\&A, 50, 491

Qu, Y., Di Matteo, P., Lehnert, M. D., \& van Driel, W. 2011, A\&A, 530, A10 Qu, Z., \& Bregman, J. N. 2018, ApJ, 856, 5

Rubin, K. H. R., Prochaska, J. X., Koo, D. C., \& Phillips, A. C. 2012, ApJ, 747, L26

Rubin, K. H. R., Prochaska, J. X., Koo, D. C., et al. 2014, ApJ, 794, 156

Savage, B. D., Kim, T.-S., Wakker, B. P., et al. 2014, ApJS, 212, 8

Schaye, J., Crain, R. A., Bower, R. G., et al. 2015, MNRAS, 446, 521

Sheinis, A. I., Bolte, M., Epps, H. W., et al. 2002, PASP, 114, 851

Shen, S., Madau, P., Aguirre, A., et al. 2012, ApJ, 760, 50

Simard, L., Willmer, C. N. A., Vogt, N. P., et al. 2002, ApJS, 142, 1

Steidel, C. C., Kollmeier, J. A., Shapley, A. E., et al. 2002, ApJ, 570, 526

Stern, J., Hennawi, J. F., Prochaska, J. X., \& Werk, J. K. 2016, ArXiv e-prints

Stewart, K. R., Kaufmann, T., Bullock, J. S., et al. 2011, ApJ, 738, 39

Stocke, J. T., Keeney, B. A., Danforth, C. W., et al. 2013, ApJ, 763, 148

Strickland, D. K., \& Heckman, T. M. 2009, ApJ, 697, 2030

Suresh, J., Rubin, K. H. R., Kannan, R., et al. 2017, Monthly Notices of the Royal Astronomical Society, 465, 2966

Trujillo-Gomez, S., Klypin, A., Primack, J., \& Romanowsky, A. J. 2011, ApJ, 742, 16

Tumlinson, J., Peeples, M. S., \& Werk, J. K. 2017, ARA\&A, 55, 389

Tumlinson, J., Thom, C., Werk, J. K., et al. 2011, Science, 334, 948 -. 2013, ApJ, 777, 59

van de Voort, F. 2017, in Astrophysics and Space Science Library, Vol. 430,

Gas Accretion onto Galaxies, ed. A. Fox \& R. Davé, 301

Werk, J. K., Prochaska, J. X., Thom, C., et al. 2013, ApJS, 204, 17

Werk, J. K., Prochaska, J. X., Cantalupo, S., et al. 2016, ApJ, 833, 54

Wolf, C., Meisenheimer, K., Rix, H.-W., et al. 2003, A\&A, 401, 73

Zahedy, F. S., Chen, H.-W., Johnson, S. D., et al. 2019, MNRAS, 484, 2257 